Modelling, Analysis and Simulation

J. Němcová 
Centrum Wiskunde \& Informatica (CWI) is the national research institute for Mathematics and Computer Science. It is sponsored by the Netherlands Organisation for Scientific Research (NWO).

$\mathrm{CWI}$ is a founding member of ERCIM, the European Research Consortium for Informatics and Mathematics.

CWI's research has a theme-oriented structure and is grouped into four clusters. Listed below are the names of the clusters and in parentheses their acronyms.

Probability, Networks and Algorithms (PNA)

Software Engineering (SEN)

\section{Modelling, Analysis and Simulation (MAS)}

Information Systems (INS)

Copyright (C) 2008, Centrum Wiskunde \& Informatica

P.O. Box 94079, 1090 GB Amsterdam (NL)

Kruislaan 413, 1098 SJ Amsterdam (NL)

Telephone +31205929333

Telefax +31205924199

ISSN 1386-3703 


\title{
Structural identifiability of polynomial and rational systems
}

\begin{abstract}
Identifiability is a property of a parametrization of a system. A parametrization is a map from a parameter set to a parametrized system. It maps parameter values to systems derived from the parametrized system by substituting the parameter values to the parameters. We say that a parametrization is globally identifiable if the parameter values can be uniquely determined from the data which are modeled by the parametrized system. It is structurally identifiable if this holds for almost all parameter values. Therefore verifying structural or global identifiability of a parametrization preceeds determination of numerical values of the parameters. In this paper, we derive necessary and sufficient conditions for the parametrizations of parametrized polynomial and parametrized rational systems to be structurally or globally identifiable. The results are applied to study the identifiability properties of the systems modeling certain biological phenomena.
\end{abstract}

2000 Mathematics Subject Classification: 93B30,93B27,93B15

Keywords and Phrases: Structural identifiability; Polynomial systems; Rational systems; Algebraic approaches; Realization theory.

Note: This work was carried out under project NWO.EW.MATH. 



\title{
Structural identifiability of polynomial and rational systems
}

\author{
Jana Němcová \\ Centrum Wiskunde $\mathcal{E}$ Informatica (CWI) \\ Amsterdam, The Netherlands
}

\begin{abstract}
Identifiability is a property of a parametrization of a system. A parametrization is a map from a parameter set to a parametrized system. It maps parameter values to systems derived from the parametrized system by substituting the parameter values to the parameters. We say that a parametrization is globally identifiable if the parameter values can be uniquely determined from the data which are modeled by the parametrized system. It is structurally identifiable if this holds for almost all parameter values. Therefore verifying structural or global identifiability of a parametrization preceeds determination of numerical values of the parameters. In this paper, we derive necessary and sufficient conditions for the parametrizations of parametrized polynomial and parametrized rational systems to be structurally or globally identifiable. The results are applied to study the identifiability properties of the systems modeling certain biological phenomena.
\end{abstract}

Key words: Structural identifiability, Polynomial systems, Rational systems, Algebraic approaches, Realization theory.

\section{Introduction}

System identification is a research topic dealing with the problem of determining a system modeling in a particular best way a certain phenomenon. In this paper we treat the identifiability problem which is one of many problems appearing in system identification.

To model a certain phenomenon we first deal with the modeling issues like experiment design, data collection, and choosing the model structure. After these

Email address: J.Nemcova@cwi.nl (Jana Němcová). 
steps we consider the phenomenon to be characterized by the collected data (usually in the form of time series) and to be modeled by a parametrized system. The parameters of the parametrized system are the uncertainties which have to be specified to get a fully specified model. Here we come to the identifiability problem. The parameter values can be determined uniquely from the data only if the parametrization is identifiable. Therefore it makes sense first to check whether the parametrization is identifiable and only then, if it holds, continue with determining or estimating the numerical values of parameters. Consequently we validate the final system modeling the data. If the parametrization is not identifiable or if the final system is not faithfully modeling the data then we have to go back to the preceeding steps. Until we derive the desired system we iterate this procedure with different experiment designs and with choosing different model structures and different methods for determining the parameters. The problems of system identification have been studied for the classes of stochastic and deterministic, linear and nonlinear, discrete-time and continuous-time systems, and for time-invariant and time-dependent (time-varying) parameters.

In this paper, attention is restricted to the problem of identifiability for deterministic continuous-time parametrized systems whose dynamics is given by polynomial or rational vector fields and whose output function is componentwise given by a polynomial or a rational function of state variables and parameters. These classes of systems are used to model gene expression, metabolic networks, and enzyme catalyzed reactions [19]. There are many approaches to study identifiability of the systems, for example the approach based on a power series expansion of the output [28], differential algebra [21], generating series approach [35], and similarity transformation method [31]. Our approach, which is related to similarity transformation or state isomorphism approach $[7,8,27,31-33,36]$, strongly relies on the results of realization theory for polynomial and rational systems. Realization theory provides a characterization of all systems (realizations) within a certain class of systems which have the same input-output behaviour as a considered parametrized system modeling the considered phenomenon. By restricting these realizations to the set of systems having the same structure as the parametrized system, we get all numerical values of parameters which by substitution to the parametrized system result in systems modeling the same data.

Many concepts of identifiability of certain parametrized systems are present in the literature. We consider the problem of structural and global identifiability of the parametrizations of parametrized polynomial and parametrized rational systems. The first paper introducing the concept of structural identifiability in system theoretic framework is [6]. Structural and algebraic identifiability for a class of nonlinear systems is studied also in [37]. These authors work in a linear algebraic setting which is related to differential algebraic approach. The meromorphic systems they consider are more general than the rational 
systems we consider. On the other hand, their assumption on the continuous differentiability up to some order of the inputs is stronger than our assumption of considering piecewise-constant inputs. Other papers dealing with identifiability of polynomial and rational systems without inputs are $[11,13]$. Structural identifiability of the models described by input-output relations (differential-algebraic expression) rather than by state-space forms is studied in differential algebraic setting in [21]. For the application of this approach to a real life problem see [15].

The outline of the paper is as follows. Section 2 introduces the concepts of polynomial and rational systems as they are developed in $[4,5,26]$ and the relevant references therein. The definitions of algebraic controllability, algebraic and rational observability are recalled. In Section 3 we define the concepts of parametrized polynomial and parametrized rational systems, parametrized systems distinguishing parameters, and structured polynomial and structured rational systems. We derive the notion of structural algebraic controllability, structural algebraic observability, and structural canonicity by a slight modification of the corresponding notions for polynomial and rational systems without parameters. The problem of global and structural identifiability of the parametrizations of parametrized polynomial and parametrized rational systems is formulated in Section 4. We characterize global and structural identifiability of the parametrizations for parametrized polynomial systems in Section 4.1. The characterization for parametrized rational systems is derived in Section 4.2. In both sections we illustrate the obtained results by a simple example. Section 5 deals with the study of structural identifiability for three parametrized systems modeling certain biological phenomena. The first example treats a model of a chain of two enzyme-catalyzed irreversible reactions. The second example considers a two-compartment model with Michaelis-Menten kinetics which is studied already in [22]. The third example deals with a system modeling peptide chain elongation. The last section concludes the paper.

\section{Polynomial and rational systems}

Within the paper we recall some basic terminology and facts of commutative algebra and algebraic geometry which are used afterwards. For more details on these topics see for example $[10,20,38]$.

Let $X \subseteq \mathbb{R}^{n}$ be an irreducible real affine variety, i.e. $X$ is determined by finitely many polynomials $f_{1}, \ldots, f_{N} \in \mathbb{R}\left[X_{1}, \ldots, X_{n}\right]$ such that $X=\left\{\left(x_{1}, \ldots, x_{n}\right) \in\right.$ $\left.\mathbb{R}^{n} \mid f_{1}\left(x_{1}, \ldots, x_{n}\right)=\cdots=f_{N}\left(x_{1}, \ldots, x_{n}\right)=0\right\}$. Irreducibility of $X$ refers to the property that $X$ cannot be written as an union of two non-empty varieties. By a polynomial on $X$ we mean a map $p: X \rightarrow \mathbb{R}$ for which there exists 
a polynomial $p^{\prime} \in \mathbb{R}\left[X_{1}, \ldots, X_{n}\right]$ such that $p=p^{\prime}$ on $\mathrm{X}$. Let $I$ denote the ideal of all polynomials of $\mathbb{R}\left[X_{1}, \ldots, X_{n}\right]$ vanishing on $X$. It is generated by $f_{1}, \ldots, f_{N}$. The set $A$ of all polynomials on $X$ is isomorphic to the quotient $\operatorname{ring} \mathbb{R}\left[X_{1}, \ldots, X_{n}\right] / I$. Since $X$ is an irreducible variety, $A$ is an integral domain which allows us to define the field $Q$ of quotients of $A$. We call the elements of $Q$ the rational functions on $X$. Note that the rational functions on $X$ do not have to be defined on all of $X$.

On $\mathbb{R}^{n}$ spaces we consider the Zariski topology so that the closed sets are defined as real affine varieties. We refer to an open/closed/dense set in Zariski topology as to a $Z$-open $/ Z$-closed/ $Z$-dense set.

To introduce polynomial and rational systems we follow Bartosiewicz's definitions in $[4,5]$. His approach generalizes the common approach of nonlinear control theory from the geometric viewpoint since the state spaces he considers are not necessarily smooth affine varieties.

By an input space $U$ we mean an arbitrary set of input values $U \subseteq \mathbb{R}^{m}$. The output space is considered to be $\mathbb{R}^{r}$. The following definition of polynomial and rational systems is a slight modification of the definition in [5, Section 2] for polynomial systems, and of [4, Definition 2] for rational systems.

Definition 2.1 A polynomial (rational) system $\Sigma$ is a quadruple $\left(X, f, h, x_{0}\right)$ where

(i) $X \subseteq \mathbb{R}^{n}$ is an irreducible real affine variety,

(ii) $f=\left\{f_{\alpha} \mid \alpha \in U\right\}$ is a family of polynomial (rational) vector fields on $X$, i.e. $f_{\alpha}=\sum_{i=1}^{n} f_{\alpha, i} \frac{\partial}{\partial X_{i}}$ where $f_{\alpha, i} \in A\left(f_{\alpha, i} \in Q\right)$ for all $\alpha \in U$ and all $i=1, \ldots, n$,

(iii) $h: X \rightarrow \mathbb{R}^{r}$ is an output map with polynomial (rational) components, thus $h_{j} \in A\left(h_{j} \in Q\right)$ for all $j=1, \ldots, r$,

(iv) $x_{0} \in X$ is an initial state of $\Sigma$ (For rational systems $x_{0}$ is such that all components of $h$ and all of the vector fields $f_{\alpha}, \alpha \in U$ are defined at $x_{0}$. By $f_{\alpha}$ being defined at $x_{0}$ we mean that $f_{\alpha}\left(O_{x_{0}}\right) \subseteq O_{x_{0}}$ where $O_{x_{0}}=\left\{\frac{g_{1}}{g_{2}} \mid g_{1}, g_{2} \in\right.$ $\left.A, g_{2}\left(x_{0}\right) \neq 0\right\}$. For polynomial systems all components of $h$ and all vector fields $f_{\alpha}, \alpha \in U$ are defined at any $x_{0} \in X$.).

As the space of input functions we consider the set $\mathcal{U}_{p c}$ of piecewise constant functions $u:\left[0, T_{u}\right) \rightarrow U$ where $T_{u} \geq 0$ depends on $u$. So, if $u \in \mathcal{U}_{p c}$ then $u=\left(\alpha_{1}, t_{1}\right)\left(\alpha_{2}, t_{2}\right) \ldots\left(\alpha_{n_{u}}, t_{n_{u}}\right)$ which means that for $t \in\left[\sum_{j=0}^{i} t_{j}, \sum_{j=0}^{i+1} t_{j}\right)$ the input $u(t)=\alpha_{i+1} \in U$ for $i=0,1, \ldots, n_{u}-1, t_{0}=0$. We assume that $n_{u}<\infty$. Every input function $u \in \mathcal{U}_{p c}$ has a time domain $\left[0, T_{u}\right)$ where $T_{u}=\sum_{j=1}^{n_{u}} t_{j}$. The restriction of an input $u$ to a shorter time domain $[0, t), t<T_{u}$ is denoted by $u_{[0, t)}$. The empty input $e$ is such input that $T_{e}=0$. Note that an input $u \in \mathcal{U}_{p c}$ can be represented in different ways as a sequence of tuples $(\alpha, t)$ with 
$\alpha \in U, t \in[0, \infty)$. For example, $u=\left(\alpha_{1}, t_{1}\right)\left(\alpha_{1}, t_{2}\right)\left(\alpha_{2}, 0\right)\left(\alpha_{3}, t_{3}\right)=\left(\alpha_{1}, t_{1}+\right.$ $\left.t_{2}\right)\left(\alpha_{3}, t_{3}\right)$. We consider all these representations to be equivalent.

Let $\Sigma=\left(X, f, h, x_{0}\right)$ be a polynomial or a rational system. Consider a constant input $u=\left(\alpha, T_{u}\right) \in \mathcal{U}_{p c}$. A trajectory of $\Sigma$ corresponding to the input $u$ is the trajectory of a vector field $f_{\alpha}$ from a point $x_{0} \in X$ restricted to the time-domain $\left[0, T_{u}\right)$, i.e. it is the map $x\left(\cdot ; x_{0}, u\right):\left[0, T_{u}\right) \rightarrow X$ such that $\frac{d}{d t}(\varphi \circ x)\left(t ; x_{0}, u\right)=\left(f_{\alpha} \varphi\right)\left(x\left(t ; x_{0}, u\right)\right)$ and $x(0)=x_{0}$ for $t \in\left[0, T_{u}\right)$ and for $\varphi \in A$. According to [5, Theorem 1] for any polynomial vector field $f$ on a variety $X$ and any $x_{0} \in X$ there exists an unique trajectory of $f$ from $x_{0}$ defined on maximal interval $[0, T)$. The corresponding result for any rational vector field $f$ on a variety $X$ and any $x_{0} \in X$ at which $f$ is defined can be found in [4]. Therefore, the trajectory of a polynomial or a rational system $\Sigma$ for an input $u=\left(\alpha, T_{u}\right) \in \mathcal{U}_{p c}$ is defined if $f_{\alpha}$ is defined at $x_{0}$ and if $T_{u} \leq T$ for $T$ determining the maximal interval $[0, T)$ on which the trajectory of $f_{\alpha}$ is defined. The trajectory of a system $\Sigma$ with an initial state $x_{0} \in X$ is for the empty input $e$ equal to $x_{0}$, i.e. $x\left(0 ; x_{0}, e\right)=x_{0}$. Let us consider an input $u=\left(\alpha_{1}, t_{1}\right) \ldots\left(\alpha_{n_{u}}, t_{n_{u}}\right) \in \mathcal{U}_{p c}$. By a trajectory of $\Sigma$ for the input $u$ we mean the map $x\left(\cdot ; x_{0}, u\right):\left[0, T_{u}\right) \rightarrow X$ such that $x\left(t ; x_{0}, u\right)=x_{i}\left(t-\sum_{j=1}^{i-1} t_{j} ; x_{0}^{i-1},\left(\alpha_{i}, t_{i}\right)\right)$ for $t \in\left[\sum_{j=1}^{i-1} t_{j}, \sum_{j=1}^{i} t_{j}\right), i=1, \ldots, n_{u}$, where $x_{0}^{i-1}=x_{i-1}\left(\sum_{j=0}^{i-1} t_{j} ; x_{0}^{i-2},\left(\alpha_{i-1}, t_{i-1}\right)\right)$ for $i=2, \ldots, n_{u}$ and $x_{0}^{0}=x_{0}$. The maps $x_{i}\left(\cdot ; x_{0}^{i-1},\left(\alpha_{i}, t_{i}\right)\right):\left[0, t_{i}\right) \rightarrow X, i=1, \ldots, n_{u}$ are the trajectories of the vector fields $f_{\alpha_{i}}$ from the points $x_{0}^{i-1}$.

Since a trajectory of a polynomial or a rational system $\Sigma=\left(X, f, h, x_{0}\right)$ does not need to exist for every input $u \in \mathcal{U}_{p c}$, we define the set $\widetilde{\mathcal{U}_{p c}}(\Sigma)$ of admissible inputs for the system $\Sigma$ as a subset of the set of input functions $\mathcal{U}_{p c}$ for which there exists a trajectory of $\Sigma$.

Definition 2.2 [24] A polynomial/rational system $\Sigma=\left(X, f, h, x_{0}\right)$ is said to be algebraically controllable (from the initial state $x_{0}$ ) if the reachable set from $x_{0}$,

$$
\mathcal{R}\left(x_{0}\right)=\left\{x\left(T_{u} ; x_{0}, u\right) \in X \mid u \in \widetilde{\mathcal{U}_{p c}}(\Sigma)\right\},
$$

is $Z$-dense in $X$.

Definition 2.3 [5] Let $\Sigma=\left(X, f=\left\{f_{\alpha} \mid \alpha \in U\right\}, h, x_{0}\right)$ be a polynomial system and let $A$ denote the algebra of polynomial functions on $X$. The observation algebra $A_{o b s}(\Sigma)$ of $\Sigma$ is the smallest subalgebra of $A$ containing all components $h_{i}, i=1, \ldots, r$ of $h$, and which is closed with respect to the derivations given by the vector fields $f_{\alpha}, \alpha \in U$. The system $\Sigma$ is called algebraically observable if $A_{o b s}(\Sigma)=A$.

Definition 2.4 [4] Let $\Sigma=\left(X, f=\left\{f_{\alpha} \mid \alpha \in U\right\}, h, x_{0}\right)$ be a polynomial or a rational system and let $Q$ denote the field of rational functions on $X$. The observation algebra $A_{o b s}(\Sigma)$ of $\Sigma$ is the smallest subalgebra of the field $Q$ containing all components $h_{i}, i=1, \ldots, r$ of $h$, and which is closed with respect 
to the derivations given by the vector fields $f_{\alpha}, \alpha \in U$. The observation field $Q_{o b s}(\Sigma)$ of the system $\Sigma$ is the field of quotients of $A_{\text {obs }}(\Sigma)$. The system $\Sigma$ is called rationally observable if $Q_{o b s}(\Sigma)=Q$.

If a polynomial system is algebraically observable then it is also rationally observable. On the other hand, there exist rationally observable polynomial systems which are not algebraically observable, see Example 2.5. For more details on algebraic and rational observability and algebraic controllability of polynomial and rational systems see $[2-5,24]$ and others.

Example 2.5 Let $\Sigma=\left(X, f=\left\{f_{\alpha} \mid \alpha \in \mathbb{R}\right\}, h, x_{0}\right)$ be a polynomial system given as $X=\mathbb{R}, f_{\alpha}=\alpha x^{2} \frac{\partial}{\partial x}$ for $\alpha \in \mathbb{R}, h=x^{2}, x_{0}=1$. By simple calculation we derive the observation algebra of $\Sigma$,

$$
A_{\text {obs }}(\Sigma)=\mathbb{R}\left[x^{2}, x^{3}, x^{4}, \ldots\right] \subsetneq \mathbb{R}[x]=A .
$$

Therefore, according to Definition 2.3, $\Sigma$ is not algebraically observable. On the other hand, for the observation field of $\Sigma$ it holds that $Q_{\text {obs }}(\Sigma)=\mathbb{R}(x)=Q$ and consequently, from Definition 2.4, the system $\Sigma$ is rationally observable.

\section{$3 \quad$ Parametrized and structured systems}

By choosing a model structure in the modeling step of system identification procedure we specify a system with parameters which are not fully determined. Depending on the modeling techniques, the parameters could have a physical or a biological meaning relevant for further investigation of the studied phenomenon. Below we introduce the concept of parametrized polynomial and parametrized rational systems. We also introduce the concept of structured polynomial and structured rational systems which are intuitively parametrized systems having the same structure.

Let $P \subseteq \mathbb{R}^{l}, l \in \mathbb{N}$ denote a parameter set which is assumed to be an irreducible real affine variety. Then $P$ is determined by the ideal $I^{P} \subseteq \mathbb{R}\left[P_{1}, \ldots, P_{l}\right]$ such that $I^{P}=\left(f_{1}^{P}, \ldots, f_{n_{P}}^{P}\right)$ for $f_{i}^{P} \in \mathbb{R}\left[P_{1}, \ldots P_{l}\right], i=1, \ldots, n_{P}<\infty$. Consider an arbitrary irreducible real affine variety $X \subseteq \mathbb{R}^{n}$ determined by the ideal $I \subseteq$ $\mathbb{R}\left[X_{1}, \ldots, X_{n}\right]$ where $I=\left(f_{1}^{X}, \ldots, f_{n_{X}}^{X}\right)$ for $f_{i}^{X} \in \mathbb{R}\left[X_{1}, \ldots X_{n}\right], i=1, \ldots, n_{X}$. Because both varieties $P$ and $X$ can be considered varieties in $\mathbb{R}^{n+l}$ and because the union of two affine varieties is an affine variety, it follows that $X \cup P \subseteq$ $\mathbb{R}^{n+l}$ is the variety determined by the ideal $I^{X \cup P}=\left(f_{i}^{X} f_{j}^{P}: 1 \leq i \leq n_{x}, 1 \leq\right.$ $\left.j \leq n_{P}\right)$. We call the elements of $A^{X \cup P} \cong \mathbb{R}\left[X_{1}, \ldots, X_{n}, P_{1}, \ldots, P_{l}\right] / I^{X \cup P}$ the polynomials on $X$ parametrized by $P$. Hence, a parametrized polynomial on $X$ parametrized by $P$ is a map $w: X \cup P \rightarrow \mathbb{R}$ for which there exists a polynomial $w^{\prime} \in \mathbb{R}\left[X_{1}, \ldots, X_{n}, P_{1}, \ldots, P_{l}\right]$ such that $w=w^{\prime}$ on $X \cup P$. 
The polynomials parametrized by a parameter $p=\left(p_{1}, \ldots, p_{l}\right) \in P$ are the elements of $A^{p} \cong \mathbb{R}\left[X_{1}, \ldots, X_{n}, p_{1}, \ldots, p_{l}\right] / I$, i.e. the elements of $A^{X \cup P}$ evaluated for $P_{1}=p_{1}, \ldots, P_{l}=p_{l}$. We will consider the situations when for different values of parameters we consider even different varieties $X$. We express the dependence of the varieties $X$ on the parameters $p \in P$ by using the notation $X^{p}$. Note that $X^{p}$ does not depend on $p$ explicitly and that it is still allowed that for different $p, p^{\prime} \in P$ the varieties $X^{p}$ and $X^{p^{\prime}}$ are the same. Every irreducible real affine variety $X^{p} \subseteq \mathbb{R}^{n_{p}}, p \in P$ is determined by the ideal $I^{p} \subseteq \mathbb{R}\left[X_{1}, \ldots, X_{n_{p}}\right]$. The parametrized polynomials on $X^{p}$ by $p=\left(p_{1}, \ldots, p_{l}\right) \in P$ are the elements of $A^{p} \cong \mathbb{R}\left[X_{1}, \ldots, X_{n_{p}}, p_{1}, \ldots, p_{l}\right] / I^{p}$. The parametrized rational functions on $X^{p}$, denoted by $Q^{p}$, are the elements of the quotient field of $A^{p}$. Since $A^{p}$ is an integral domain, $Q^{p}$ is defined.

Definition 3.1 Let a parameter set $P \subseteq \mathbb{R}^{l}$ be an irreducible real affine variety. By a parametrized polynomial (rational) system $\Sigma(P)$ we mean a family of polynomial (rational) systems $\{\Sigma(p) \mid p \in P\}$ such that $\Sigma(p)=\left(X^{p}, f^{p}, h^{p}, x_{0}^{p}\right)$ where

(i) $X^{p} \subseteq \mathbb{R}^{n_{p}}$ is an irreducible real affine variety,

(ii) $f^{p}=\left\{f_{\alpha}^{p} \mid \alpha \in U\right\}$ where $f_{\alpha}^{p}=\sum_{i=1}^{n_{p}} f_{\alpha, i}^{p} \frac{\partial}{\partial X_{i}}$ and $f_{\alpha, i}^{p} \in A^{p}\left(f_{\alpha, i}^{p} \in Q^{p}\right)$ for all $\alpha \in U$ and all $i=1, \ldots, n_{p}$,

(iii) $h^{p}: X^{p} \rightarrow \mathbb{R}^{r}$ is an output map with the components $h_{j}^{p} \in A^{p}\left(h_{j}^{p} \in Q^{p}\right)$, $j=1, \ldots, r$,

(iv) $x_{0}^{p}=\left(x_{1}^{p}(0), \ldots, x_{n_{p}}^{p}(0)\right) \in X^{p}$ where $x_{i}^{p}(0) \in \mathbb{R}\left[p_{1}, \ldots, p_{l}\right]$ for $i=1, \ldots, n_{p}$ (for a parametrized rational system $x_{i}^{p}(0) \in \mathbb{R}\left(p_{1}, \ldots, p_{l}\right)$ for $i=1, \ldots, n_{p}$, and all components of $h^{p}$ and all of $f_{\alpha}^{p}, \alpha \in U$ are defined at $\left.x_{0}^{p}\right)$.

We assume that the systems $\Sigma(p), p \in P$ have the same input spaces $U$ and the same output spaces $\mathbb{R}^{r}$. The map $\mathcal{P}: P \rightarrow \Sigma(P)$ defined as $\mathcal{P}(p)=\Sigma(p)$ for $p \in P$ is called the parametrization of $\Sigma(P)$.

Example 3.2 As an example of a parametrized rational system consider a family $\Sigma(P)$ of rational systems $\Sigma(p), p \in P=\mathbb{R}^{4}$ modeling a chain of two enzyme-catalyzed irreversible reactions. The systems $\Sigma(p)$ of the family $\Sigma(P)$ are of the following form:

$$
\begin{aligned}
X^{p} & =\mathbb{R}^{2}, \\
f_{\alpha}^{p} & =\left(-\frac{p_{1} x_{1}}{p_{2}+x_{1}}+\alpha\right) \frac{\partial}{\partial x_{1}}+\left(\frac{p_{1} x_{1}}{p_{2}+x_{1}}-\frac{p_{3} x_{2}}{p_{4}+x_{2}}\right) \frac{\partial}{\partial x_{2}}, \alpha \in U, \\
h^{p}\left(x_{1}, x_{2}\right) & =\left(h_{1}^{p}\left(x_{1}, x_{2}\right), h_{2}^{p}\left(x_{1}, x_{2}\right)\right)=\left(\frac{p_{1} x_{1}}{p_{2}+x_{1}}, \frac{p_{3} x_{2}}{p_{4}+x_{2}}\right), \\
x_{1}^{p}(0) & =1, \quad x_{2}^{p}(0)=1 .
\end{aligned}
$$

This parametrized system $\Sigma(P)$ is discussed further in Example 5.1.

Let $P \subseteq \mathbb{R}^{l}$ be a parameter set determined by the ideal $I^{P} \subseteq \mathbb{R}\left[P_{1}, \ldots, P_{l}\right]$. 
The polynomials on $P$ are denoted by $A^{P} \cong \mathbb{R}\left[P_{1}, \ldots, P_{l}\right] / I^{P}$. Let $\Sigma(P)$ be a parametrized polynomial system and let $\Sigma(p)=\left(X^{p} \subseteq \mathbb{R}^{n_{p}}, f^{p}=\left\{f_{\alpha}^{p}=\right.\right.$ $\left.\left.\sum_{i=1}^{n_{p}} f_{\alpha, i}^{p} \frac{\partial}{\partial X_{i}} \mid \alpha \in U\right\}, h^{p}, x_{0}^{p}\right) \in \Sigma(P)$ be a polynomial system determined by a parameter $p=\left(p_{1}, \ldots, p_{l}\right) \in P$. Let $\varphi_{1}, \ldots, \varphi_{n_{p}} \in A^{p}$ be the polynomials on $X^{p}$ corresponding to the polynomials $X_{1}, \ldots, X_{n_{p}} \in \mathbb{R}\left[X_{1}, \ldots, X_{n_{p}}\right]$. Then $\mathbb{R}\left[\varphi_{1}, \ldots, \varphi_{n_{p}}\right]=A^{p}$ and the elements $f_{\alpha, i}^{p}, h_{j}^{p}, x_{i}^{p}(0) \in A^{p}$ for $i=1, \ldots, n_{p}, j=$ $1, \ldots, r$, and $\alpha \in U$, can be written in the form:

$$
\begin{aligned}
f_{\alpha, i}^{p} & =\sum_{a_{1}, \ldots, a_{n_{p}} \in \mathbb{N}} q_{i ; a_{1}, \ldots, a_{n_{p}}}^{f_{\alpha}}\left(p_{1}, \ldots, p_{l}\right) \varphi_{1}^{a_{1}} \ldots \varphi_{n_{p}}^{a_{n_{p}}}, \\
h_{j}^{p} & =\sum_{b_{1}, \ldots, b_{n_{p}} \in \mathbb{N}} q_{j ; b_{1}, \ldots, b_{n_{p}}}^{h}\left(p_{1}, \ldots, p_{l}\right) \varphi_{1}^{b_{1}} \ldots \varphi_{n_{p}}^{b_{n_{p}}}, \\
x_{i}^{p}(0) & =q_{i}^{x_{0}}\left(p_{1}, \ldots, p_{l}\right),
\end{aligned}
$$

where $q_{i ; a_{1}, \ldots, a_{n_{p}}}^{f_{\alpha}}, q_{j ; b_{1}, \ldots, b_{n_{p}}}^{h}, q_{i}^{x_{0}} \in \mathbb{R}\left[P_{1}, \ldots, P_{l}\right]$ are such that for all $i=1, \ldots, n_{p}$ only finitely many $\left(a_{1}, \ldots, a_{n_{p}}\right) \in \mathbb{N}^{n_{p}}$ are such that $q_{i ; a_{1}, \ldots, a_{n_{p}}}^{f_{\alpha}}\left(p_{1}, \ldots, p_{l}\right) \neq 0$ and for all $j=1, \ldots, r$ only finitely many $\left(b_{1}, \ldots, b_{n_{p}}\right) \in \mathbb{N}^{n_{p}}$ are such that $q_{j ; b_{1}, \ldots, b_{n_{p}}}^{h}\left(p_{1}, \ldots, p_{l}\right) \neq 0$.

Definition 3.3 Let $P \subseteq \mathbb{R}^{l}$ be a parameter set and let $\Sigma(P)$ be a parametrized polynomial system. We say that $\Sigma(P)=\left\{\Sigma(p)=\left(X^{p} \subseteq \mathbb{R}^{n_{p}}, f^{p}=\left\{f_{\alpha}^{p}=\right.\right.\right.$ $\left.\left.\left.\sum_{i=1}^{n_{p}} f_{\alpha, i}^{p} \frac{\partial}{\partial X_{i}} \mid \alpha \in U\right\}, h^{p}, x_{0}^{p}\right) \mid p \in P\right\}$ is distinguishing parameters if for every $p \in P$ the polynomials $q_{i ; a_{1}, \ldots, a_{n_{p}}}^{f_{\alpha}}, q_{j ; b_{1}, \ldots, b_{n_{p}}}^{h}, q_{i}^{x_{0}} \in \mathbb{R}\left[P_{1}, \ldots, P_{l}\right], i=1, \ldots, n_{p}$, $j=1, \ldots, r, \alpha \in U$ determined by the equalities (1), (2), (3) distinguish the points of the variety $P$ (we say that for all $p \in P$ the system $\Sigma(p)$ is distinguishing parameters), i.e. if for all $p \in P$ it holds that $\mathbb{R}\left[\left\{q_{i ; a_{1}, \ldots, a_{n_{p}}}^{f_{\alpha}}, q_{j ; b_{1}, \ldots, b_{n_{p}}}^{h}\right.\right.$, $\left.\left.q_{i}^{x_{0}} \mid i=1, \ldots, n_{p}, j=1, \ldots, r, \alpha \in U\right\}\right] \cong \mathbb{R}\left[P_{1}, \ldots, P_{l}\right] / I^{P} \cong A^{P}$.

Let $P \subseteq \mathbb{R}^{l}$ be a parameter set determined by the ideal $I^{P} \subseteq \mathbb{R}\left[P_{1}, \ldots, P_{l}\right]$. The polynomials on $P$ are denoted by $A^{P} \cong \mathbb{R}\left[P_{1}, \ldots, P_{l}\right] / I^{P}$ and the set of rational functions on $P$, defined as the field of fractions of $A^{P}$, is denoted by $Q^{P}$. Let $\Sigma(P)$ be a parametrized rational system and let $\Sigma(p)=\left(X^{p} \subseteq\right.$ $\left.\mathbb{R}^{n_{p}}, f^{p}=\left\{f_{\alpha}^{p}=\sum_{i=1}^{n_{p}} f_{\alpha, i}^{p} \frac{\partial}{\partial X_{i}} \mid \alpha \in U\right\}, h^{p}, x_{0}^{p}\right) \in \Sigma(P)$ be a rational system determined by a parameter $p=\left(p_{1}, \ldots, p_{l}\right) \in P$. For the elements $f_{\alpha, i}^{p}, h_{j}^{p} \in Q^{p}$, $i=1, \ldots, n_{p}, j=1, \ldots, r, \alpha \in U$ there exist $f_{\alpha, i, n u m}^{p}, f_{\alpha, i, \text { den }}^{p}, h_{j, \text { num }}^{p}, h_{j, \text { den }}^{p} \in A^{p}$ such that $f_{\alpha, i}^{p}=\frac{f_{\alpha, i, n u m}^{p}}{f_{\alpha, i, d e n}^{p}}$ and $h_{j}^{p}=\frac{h_{j, \text { num }}^{p}}{h_{j, \text { den }}^{p}}$. Let $\varphi_{1}, \ldots, \varphi_{n_{p}} \in A^{p}$ be the polynomials on $X^{p}$ corresponding to the polynomials $X_{1}, \ldots, X_{n_{p}} \in \mathbb{R}\left[X_{1}, \ldots, X_{n_{p}}\right]$. Then the elements $f_{\alpha, i}^{p}, h_{j}^{p}, x_{i}^{p}(0) \in Q^{p}$ for $i=1, \ldots, n_{p}, j=1, \ldots, r$, and $\alpha \in U$, can be written in the form:

$$
f_{\alpha, i}^{p}=\frac{f_{\alpha, i, \text { num }}^{p}}{f_{\alpha, i, \text { den }}^{p}}=\frac{\sum_{a_{1}^{1}, \ldots, a_{n_{p}}^{1} \in \mathbb{N}} q_{1, i ; a_{1}^{1}, \ldots, a_{n_{p}}^{1}}^{f_{\alpha}}\left(p_{1}, \ldots, p_{l}\right) \varphi_{1}^{a_{1}^{1}} \ldots \varphi_{n_{p}}^{a_{n_{p}}^{1}}}{\sum_{a_{1}^{2}, \ldots, a_{n_{p}}^{2} \in \mathbb{N}} q_{2, i ; a_{1}^{2}, \ldots, a_{n_{p}}^{2}}^{f_{\alpha}}\left(p_{1}, \ldots, p_{l}\right) \varphi_{1}^{a_{1}^{2}} \ldots \varphi_{n_{p}}^{a_{n_{p}}^{2}}},
$$




$$
\begin{aligned}
h_{j}^{p} & =\frac{h_{j, \text { num }}^{p}}{h_{j, \text { den }}^{p}}=\frac{\sum_{b_{1}^{1}, \ldots, b_{n_{p}}^{1} \in \mathbb{N}} q_{1, j ; b_{1}^{1}, \ldots, b_{n_{p}}^{1}}^{h}\left(p_{1}, \ldots, p_{l}\right) \varphi_{1}^{b_{1}^{1}} \ldots \varphi_{n_{p}}^{b_{n_{p}}^{1}}}{\left.\sum_{b_{1}^{2}, \ldots, b_{n_{p}}^{2} \in \mathbb{N}} q_{2, j ; b_{1}^{2}, \ldots, b_{n_{p}}^{2}}^{h}\left(p_{1}, \ldots, p_{l}\right) \varphi_{1}^{b_{1}^{2}} \ldots \varphi_{n_{p}}^{b_{n_{p}}^{2}}\right]}, \\
x_{i}^{p}(0) & =q_{i}^{x_{0}}\left(p_{1}, \ldots, p_{l}\right),
\end{aligned}
$$

where $q_{1, i ; a_{1}^{1}, \ldots, a_{n_{p}}^{1}}^{f_{\alpha}}, q_{2, i ; a_{1}^{2}, \ldots, a_{n_{p}}^{2}}^{f_{\alpha}}, q_{1, j ; b_{1}^{1}, \ldots, b_{n_{p}}^{1}}^{h}, q_{2, j ; b_{1}^{2}, \ldots, b_{n_{p}}^{2}}^{h}, q_{i}^{x_{0}} \in \mathbb{R}\left(P_{1}, \ldots, P_{l}\right)$ and all sums in (4), (5), and (6) have only finitely many summands.

Definition 3.4 Let $P \subseteq \mathbb{R}^{l}$ be a parameter set and let $\Sigma(P)$ be a parametrized rational system. We say that $\Sigma(P)=\left\{\Sigma(p)=\left(X^{p} \subseteq \mathbb{R}^{n_{p}}, f^{p}=\left\{f_{\alpha}^{p}=\right.\right.\right.$ $\left.\left.\left.\sum_{i=1}^{n_{p}} f_{\alpha, i}^{p} \frac{\partial}{\partial X_{i}} \mid \alpha \in U\right\}, h^{p}, x_{0}^{p}\right) \mid p \in P\right\}$ is distinguishing parameters if for every $p \in P$ the rational functions $q_{1, i ; a_{1}^{1}, \ldots, a_{n_{p}}^{1}}^{f_{\alpha}}, q_{2, i ; a_{1}^{2}, \ldots, a_{n_{p}}^{2}}^{f_{\alpha}}, q_{1, j ; b_{1}^{1}, \ldots, b_{n_{p}}^{1}}^{h}, q_{2, j ; b_{1}^{2}, \ldots, b_{n_{p}}^{2}}^{h}, q_{i}^{x_{0}} \in$ $\mathbb{R}\left(P_{1}, \ldots, P_{l}\right), i=1, \ldots, n_{p}, j=1, \ldots, r, \alpha \in U$ determined by the equalities (4), (5), (6) distinguish the points of the variety $P$ (we say that for all $p \in P$ the system $\Sigma(p)$ is distinguishing parameters), i.e. if for every $p \in P$ it holds that $\mathbb{R}\left(\left\{q_{1, i ; a_{1}^{1}, \ldots, a_{n_{p}}^{1}}^{f_{\alpha}}, q_{2, i ; a_{1}^{2}, \ldots, a_{n_{p}}^{2}}^{f_{\alpha}}, q_{1, j ; b_{1}^{1}, \ldots, b_{n_{p}}^{1}}^{h}, q_{2, j ; b_{1}^{2}, \ldots, b_{n_{p}}^{2}}^{h}, q_{i}^{x_{0}} \mid i=\right.\right.$ $\left.\left.1, \ldots, n_{p}, j=1, \ldots, r, \alpha \in U\right\}\right) \cong Q^{P}$.

Example 3.5 Let $\Sigma(P)=\left\{\Sigma(p)=\left(\mathbb{R}, f=\left\{f_{\alpha}=\left(p_{1}^{2}-\alpha p_{2}^{2} x\right) \frac{\partial}{\partial x} \mid \alpha \in \mathbb{R}\right\}, h=\right.\right.$ $\left.\left.1_{\mathbb{R}}, x_{0}=1\right) \mid p=\left(p_{1}, p_{2}\right) \in P=\mathbb{R}^{2}\right\}$ be a parametrized polynomial system. Consider a system $\Sigma(p) \in \Sigma(P)$ determined by a parameter $p=\left(p_{1}, p_{2}\right) \in \mathbb{R}^{2}$. The only non-zero polynomials of $\left\{q_{1 ; a_{1}}^{f_{\alpha}}, q_{1 ; b_{1}}^{h}, q_{1}^{x_{0}} \mid a_{1}, b_{1} \in \mathbb{N}\right\}$ derived by (1), (2), (3) for $\Sigma(p)$ are

$$
q_{1 ; 0}^{f_{\alpha}}\left(p_{1}, p_{2}\right)=p_{1}^{2}, q_{1 ; 1}^{f_{\alpha}}\left(p_{1}, p_{2}\right)=-\alpha p_{2}^{2}, q_{1 ; 1}^{h}\left(p_{1}, p_{2}\right)=1, q_{1}^{x_{0}}\left(p_{1}, p_{2}\right)=1 .
$$

Then $\mathbb{R}\left[q_{1 ; 0}^{f_{\alpha}}, q_{1 ; 1}^{f_{\alpha}}, q_{1 ; 1}^{h}, q_{1}^{x_{0}}\right]=\mathbb{R}\left[P_{1}^{2}, P_{2}^{2}\right] \subsetneq \mathbb{R}\left[P_{1}, P_{2}\right]$. Because $P=\mathbb{R}^{2}$ and thus $A^{P}=\mathbb{R}\left[P_{1}, P_{2}\right]$, the system $\Sigma(p)$ is not distinguishing parameters for any $p \in P$. Finally, the parametrized system $\Sigma(P)$ is not distinguishing parameters.

To introduce the concept of structured systems we first recall the notions of polynomial and rational mappings between varieties, and of isomorphic and birationally equivalent varieties. More extensive exposition can be found in $[10$, Ch.5].

Let $V_{1} \subseteq \mathbb{R}^{n_{1}}$ and $V_{2} \subseteq \mathbb{R}^{n_{2}}$ be two irreducible real affine varieties. A function $\Phi: V_{1} \rightarrow V_{2}$ is said to be a polynomial mapping from $V_{1}$ to $V_{2}$ if there exist polynomials $f_{1}, \ldots, f_{n_{2}} \in \mathbb{R}\left[X_{1}, \ldots, X_{n_{1}}\right]$ such that $\Phi\left(a_{1}, \ldots, a_{n_{1}}\right)=$ $\left(f_{1}\left(a_{1}, \ldots, a_{n_{1}}\right), \ldots, f_{n_{2}}\left(a_{1}, \ldots, a_{n_{1}}\right)\right)$ for all $\left(a_{1}, \ldots, a_{n_{1}}\right) \in V_{1}$. A function $\Phi$ : $V_{1} \rightarrow V_{2}$ is said to be a rational mapping from $V_{1}$ to $V_{2}$ if $\Phi$ can be represented as $\Phi=\left(\frac{f_{1}}{g_{1}}, \ldots, \frac{f_{n_{2}}}{g_{n_{2}}}\right)$ where $\frac{f_{i}}{g_{i}} \in \mathbb{R}\left(X_{1}, \ldots, X_{n_{1}}\right), \Phi$ is defined at some point of $V_{1}$, and for every $\left(a_{1}, \ldots, a_{n_{1}}\right) \in V_{1}$ where $\Phi$ is defined $\Phi\left(a_{1}, \ldots, a_{n_{1}}\right) \in V_{2}$. 
When we speak about a rational mapping between varieties, composition or equality of rational mappings we have to have in mind the following. A rational mapping from a variety $V_{1}$ to a variety $V_{2}$ does not have to be defined on all $V_{1}$. The composition $\Psi \circ \Phi$ of two rational mappings $\Phi: V_{1} \rightarrow V_{2}$ and $\Psi: V_{2} \rightarrow V_{3}$ is defined if there is a point $p \in V_{1}$ such that $\Phi$ is defined at $p$ and $\Psi$ is defined at $\Phi(p)$. If $\Phi, \Psi: V_{1} \rightarrow V_{2}$ are rational mappings represented as $\Phi=\left(\frac{f_{1}}{g_{1}}, \ldots, \frac{f_{n_{2}}}{g_{n_{2}}}\right), \Psi=\left(\frac{h_{1}}{k_{1}}, \ldots, \frac{h_{n_{2}}}{k_{n_{2}}}\right)$ then $\Phi=\Psi$ if $f_{i} k_{i}-h_{i} g_{i} \in I_{V_{1}}$, where $I_{V_{1}}$ is the ideal of polynomials of $\mathbb{R}\left[X_{1}, \ldots, X_{n_{1}}\right]$ vanishing on $V_{1}$, for every $i=1, \ldots, n_{1}$.

Let $V_{1} \subseteq \mathbb{R}^{n_{1}}$ and $V_{2} \subseteq \mathbb{R}^{n_{2}}$ be two irreducible real affine varieties. We say that $V_{1}$ and $V_{2}$ are isomorphic if there exist polynomial mappings $\phi: V_{1} \rightarrow V_{2}$ and $\psi: V_{2} \rightarrow V_{1}$ such that $\phi \circ \psi=1_{V_{2}}$ and $\psi \circ \phi=1_{V_{1}}$. We say that $V_{1}$ and $V_{2}$ are birationally equivalent if there exist rational mappings $\phi: V_{1} \rightarrow V_{2}$ and $\psi: V_{2} \rightarrow V_{1}$ such that $\phi \circ \psi=1_{V_{2}}$ and $\psi \circ \phi=1_{V_{1}}$.

The following definition is a slight modification of the definition of isomorphic polynomial systems in [5] and of the definition of birationally equivalent rational systems in [4].

Definition 3.6 Let $P$ be a parameter set and let $\Sigma(P)$ be a parametrized polynomial (rational) system. Recall that the systems $\Sigma(p) \in \Sigma(P)$ have the same input spaces $U$ and the same output spaces $\mathbb{R}^{r}$. Let $\Sigma(p)=\left(X^{p}, f^{p}=\right.$ $\left.\left\{f_{\alpha}^{p} \mid \alpha \in U\right\}, h^{p}, x_{0}^{p}\right)$ and $\Sigma\left(p^{\prime}\right)=\left(X^{p^{\prime}}, f^{p^{\prime}}=\left\{f_{\alpha}^{p^{\prime}} \mid \alpha \in U\right\}, h^{p^{\prime}}, x_{0}^{p^{\prime}}\right)$ be two systems of $\Sigma(P)$. We say that $\Sigma(p)$ and $\Sigma\left(p^{\prime}\right)$ are isomorphic (birationally equivalent) if

(i) the state spaces $X^{p}$ and $X^{p^{\prime}}$ are isomorphic (birationally equivalent), i.e. there exist polynomial (rational) mappings $\phi: X^{p} \rightarrow X^{p^{\prime}}, \psi: X^{p^{\prime}} \rightarrow X^{p}$ such that $\phi \circ \psi=1_{X^{p^{\prime}}}$ and $\psi \circ \phi=1_{X^{p}}$, and

(ii) $\forall \varphi \in A^{p^{\prime}}\left(Q^{p^{\prime}}\right), \forall \alpha \in U: f_{\alpha}^{p}(\varphi \circ \phi)=\left(f_{\alpha}^{p^{\prime}} \varphi\right) \circ \phi$,

(iii) $h^{p^{\prime}} \circ \phi=h^{p}$, and

(iv) $\phi$ is defined at $x_{0}^{p}$, and $\phi\left(x_{0}^{p}\right)=x_{0}^{p^{\prime}}$.

We call $\phi$ an isomorphism (a birational isomorphism).

Definition 3.7 Let $P$ be a parameter set. We say that a parametrized polynomial (rational) system $\Sigma(P)$ is a structured system if for every $\Sigma(p), \Sigma\left(p^{\prime}\right) \in$ $\Sigma(P)$ the state spaces $X^{p}, X^{p^{\prime}}$ are isomorphic (birationally equivalent) and there exist polynomial (rational) mappings $\phi: X^{p} \rightarrow X^{p^{\prime}}, \psi: X^{p^{\prime}} \rightarrow X^{p}$ such that $\phi \circ \psi=1_{X^{p^{\prime}}}, \psi \circ \phi=1_{X^{p}}$, and if we symbolically identify $p_{1}=p_{1}^{\prime}, \ldots, p_{l}=$ $p_{l}^{\prime}$ then the conditions (ii)-(iv) of Definition 3.6 are satisfied. Namely,

(i) $f_{\alpha}^{p}(\varphi \circ \phi)=\left(f_{\alpha}^{p^{\prime}} \varphi\right) \circ \phi$ for all $\alpha \in U$, and all $\varphi \in A^{p^{\prime}}\left(Q^{p^{\prime}}\right)$,

(ii) $h_{j}^{p}=h_{j}^{p^{\prime}} \circ \phi$ for all $j=1, \ldots, r$, 
(iii) $\phi$ is defined at $x_{0}^{p}$ and $\phi\left(x_{0}^{p}\right)=x_{0}^{p^{\prime}}$.

A structured system is a parametrized system such that after symbolic identification of parameters $\left(p_{1}=p_{1}^{\prime}, \ldots, p_{l}=p_{l}^{\prime}\right)$ the systems of this structured system, i.e. the systems derived as the evaluations of the structured system for all parameter values, are isomorphic (in the case of parametrized polynomial systems) or birationally equivalent (in the case of parametrized rational systems).

Note that allowing different state spaces for the systems of a structured system does not correspond to the linear case [34] where it is natural to assume that all state spaces of a structured linear system are the same.

Consider a parametrized polynomial (rational) system $\Sigma(P)$ and let us assume that the state spaces of all systems of $\Sigma(P)$ are the same. Then the state spaces $X^{p}=X^{p^{\prime}}$ for $p, p^{\prime} \in P$ are both isomorphic and birationally equivalent. The corresponding polynomial and rational mappings are the identity maps $1_{X^{p}}$ and $1_{X^{p^{\prime}}}$. Therefore, the system $\Sigma(P)$ is a structured system if after symbolic identification $p_{1}=p_{1}^{\prime}, \ldots, p_{l}=p_{l}^{\prime}$ of the parameters it follows that

(i) $f_{\alpha}^{p}=f_{\alpha}^{p^{\prime}}$ for all $\alpha \in U$,

(ii) $h_{j}^{p}=h_{j}^{p^{\prime}}$ for all $j=1, \ldots, r$,

(iii) $x_{i}^{p}(0)=x_{i}^{p^{\prime}}(0)$ for $i=1, \ldots, n_{p}=n_{p^{\prime}}$.

Any structured system is a parametrized system. The converse is not true which is demonstrated by the following example.

Example 3.8 Consider a parameter set $P=\mathbb{R}^{3}$ and consider a parametrized polynomial system $\Sigma(P)$ such that the systems of $\Sigma(P)$ are given for different parameters in the following way:

- $\Sigma(p)=\left(X^{p}, f^{p}, h^{p}, x_{0}^{p}\right)$ for $p=\left(p_{1}, p_{2}, p_{3}\right) \in P_{1}=\left\{\left(p_{1}, p_{2}, p_{3}\right) \in \mathbb{R}^{3} \mid p_{1}^{2}+\right.$ $\left.p_{2}^{2}+p_{3}^{2}=1, p_{3} \geq 0\right\}$ is given as

$$
X^{p}=\mathbb{R}, f_{\alpha}^{p}=\left(p_{1}-p_{2} x^{2}\right) \frac{\partial}{\partial x}, h^{p}=p_{3} x, x_{0}^{p}=p_{1},
$$

- $\Sigma(p)=\left(X^{p}, f^{p}, h^{p}, x_{0}^{p}\right)$ for $p=\left(p_{1}, p_{2}, p_{3}\right) \in P_{2}=\left\{\left(p_{1}, p_{2}, p_{3}\right) \in \mathbb{R}^{3} \mid p_{1}^{2}+\right.$ $\left.p_{2}^{2}+p_{3}^{2}=1, p_{3}<0\right\}$ is given as

$$
\begin{aligned}
X^{p} & =\left\{\left(x_{1}, x_{2}\right) \in \mathbb{R}^{2} \mid x_{1}-x_{2}=0\right\}, f_{\alpha}^{p}=\left(p_{1}-p_{2} x_{2}^{2}\right) \frac{\partial}{\partial x_{1}}+\left(p_{1}-p_{2} x_{1}^{2}\right) \frac{\partial}{\partial x_{2}}, \\
h^{p} & =p_{3} / 2 x_{1}+p_{3} / 2 x_{2}, x_{0}^{p}=\left(x_{1}^{p}(0), x_{2}^{p}(0)\right)=\left(p_{1}, p_{1}\right)
\end{aligned}
$$


- $\Sigma(p)=\left(X^{p}, f^{p}, h^{p}, x_{0}^{p}\right)$ for $p=\left(p_{1}, p_{2}, p_{3}\right) \in P \backslash\left\{P_{1} \cup P_{2}\right\}$ is given as

$$
X^{p}=\mathbb{R}, f_{\alpha}^{p}=\left(p_{1}-p_{2} x_{2}^{2}\right) \frac{\partial}{\partial x}, h^{p}=p_{2} x, x_{0}^{p}=p_{3} .
$$

The parametrized system $\Sigma(P)=\left\{\Sigma(p) \mid p \in \mathbb{R}^{3}\right\}$ is not a structured system. Because the polynomial mappings $\phi: \mathbb{R} \rightarrow\left\{\left(x_{1}, x_{2}\right) \in \mathbb{R}^{2} \mid x_{1}-x_{2}=0\right\}$ defined as $\phi(x)=(x, x)$ for $x \in \mathbb{R}$, and $\psi:\left\{\left(x_{1}, x_{2}\right) \in \mathbb{R}^{2} \mid x_{1}-x_{2}=0\right\} \rightarrow \mathbb{R}$ defined as $\psi\left(x_{1}, x_{2}\right)=x_{1}$ are such that $\phi \circ \psi=1_{\left\{\left(x_{1}, x_{2}\right) \in \mathbb{R}^{2} \mid x_{1}-x_{2}=0\right\}}$ and $\psi \circ \phi=1_{\mathbb{R}}$, the varieties $\mathbb{R}$ and $\left\{\left(x_{1}, x_{2}\right) \in \mathbb{R}^{2} \mid x_{1}-x_{2}=0\right\}$ are isomorphic. Moreover, $\phi$ is such that for every polynomial $\varphi$ on $\left\{\left(x_{1}, x_{2}\right) \in \mathbb{R}^{2} \mid x_{1}-x_{2}=0\right\}$ it holds that

$$
\begin{aligned}
& \left(p_{1}-p_{2} x^{2}\right) \frac{\partial}{\partial x}(\varphi \circ \phi)=\left(\left(p_{1}-p_{2} x_{2}^{2}\right) \frac{\partial}{\partial x_{1}} \varphi+\left(p_{1}-p_{2} x_{1}^{2}\right) \frac{\partial}{\partial x_{2}} \varphi\right) \circ \phi \\
& p_{3} x=\frac{p_{3}}{2} x+\frac{p_{3}}{2} x \\
& \phi\left(p_{1}\right)=\left(p_{1}, p_{1}\right) .
\end{aligned}
$$

Therefore the parametrized system $\Sigma\left(P_{1} \cup P_{2}\right)=\left\{\Sigma(p) \mid p \in\left\{\left(p_{1}, p_{2}, p_{3}\right) \in\right.\right.$ $\left.\left.\mathbb{R}^{3} \mid p_{1}^{2}+p_{2}^{2}+p_{3}^{2}=1\right\}\right\}$ is a structured system.

To derive the results of the succeeding sections we introduce the structural concepts of algebraic and rational observability, algebraic controllability, canonicity, and distinguishability of parameters.

Definition 3.9 Let $P$ be a parameter set. We say that a parametrized polynomial (rational) system $\Sigma(P)$ is

(i) structurally algebraically controllable if there exists a variety $C \subsetneq P$ such that $\Sigma(p)$ is algebraically controllable (from $x_{0}^{p}$ ) for all $p \in P \backslash C$,

(ii) structurally algebraically observable if there exists a variety $O \subsetneq P$ such that $\Sigma(p)$ is algebraically observable (rationally observable for rational systems) for all $p \in P \backslash O$,

(iii) structurally canonical if there exists a variety $C O \subsetneq P$ such that $\Sigma(p)$ is canonical (thus both algebraically observable, rationally observable for rational systems, and algebraically controllable) for all $p \in P \backslash C O$,

(iv) structurally distinguishing parameters if there exists a variety $D \subsetneq P$ such that $\Sigma(p)$ is distinguishing parameters for all $p \in P \backslash D$,

Proposition 3.10 Let $P$ be a parameter set. A parametrized polynomial (rational) system $\Sigma(P)$ is structurally canonical if and only if it is structurally algebraically controllable and structurally algebraically observable.

Proof: $\quad(\Rightarrow)$ A parametrized polynomial (rational) system which is structurally canonical is also structurally algebraically controllable and structurally 
algebraically observable. We can consider sets $C$ and $O$ to be equal to a set $C O$ which is given by structural canonicity.

$(\Leftarrow)$ Assume that $\Sigma(P)$ is structurally algebraically controllable and structurally algebraically observable. There exist varieties $C, O \subsetneq P$ such that $\Sigma(p)$ is algebraically controllable (from $x_{0}^{p}$ ) for all $p \in P \backslash C$, and $\Sigma(p)$ is algebraically (rationally for rational $\Sigma(p)$ ) observable for all $p \in P \backslash O$. We define a set $C O$ to be the union of the sets $C$ and $O$. Because the union of two varieties is a variety, the set $C O=C \cup O$ is a variety. Since $P \backslash C O \subseteq P \backslash C$ and $P \backslash C O \subseteq P \backslash O$, the system $\Sigma(p)$ for $p \in P \backslash C O$ is both algebraically (rationally) observable and algebraically controllable (from $x_{0}^{p}$ ). If $C O=P$ then the variety $P$ would be the union of two non-empty strict subvarieties of $P$, i.e. $P$ would be reducible. But this contradicts the irreducibility of $P$. Therefore $C O \subsetneq P$ and finally, $\Sigma(P)$ is structurally canonical.

Corollary 3.11 Let $\Sigma(P)$ be a parametrized polynomial (rational) system. Let $C$ and $O$ be the smallest strict subvarieties of $P$ such that $\Sigma(p)$ is algebraically controllable for all $p \in P \backslash C$ and algebraically observable (rationally observable for $\Sigma(P)$ being parametrized rational system) for all $p \in P \backslash O$. Then the variety $C O$ for which $\Sigma(p), p \in C O$ is canonical is such that $C \cup O \subseteq C O \subsetneq P$.

\section{Structural and global identifiability}

To study a certain phenomenon we observe its behaviour and apply a priori knowledge of related fields. The observation of the phenomenon consists of measuring the responses (outputs) of the studied object to stimulating signals (inputs). These measurement then characterize the phenomenon. They are usually of the form of a set of tuples $(u, y)$ where $u$ and $y$ are the functions of time with the same time domain which record the inputs (in the case of $u$ ) and outputs (in the case of $y$ ) measured for the considered phenomenon.

Consider an input $u:\left[0, T_{u}\right) \rightarrow \mathbb{R}^{m}$ and an output $y:\left[0, T_{u}\right) \rightarrow \mathbb{R}^{r}$ corresponding to $u$. The tuple $(u, y)$ provides us the same information about the phenomenon as the set of tuples $\left\{\left(u_{[0, t)}, y(t)\right) \mid t \in\left[0, T_{u}\right]\right\}$ where $u_{[0, t)}$ is the restriction of the input $u$ to the time domain $[0, t)$ and $y(t)$ is the value of the output $y$ at the time $t$ which is the end point of the time domain of the input $u_{[0, t)}$.

In this paper we assume that the measurements are provided in the form of the tuples $\left(u_{[0, t)}, y(t)\right), t \in\left[0, T_{u}\right]$ where $u$ is a stimulating input and $y$ is the response of a studied object to this input after a certain time. Moreover, we assume that the inputs which can be applied to study the considered phenomenon are given as a set of admissible inputs $\widetilde{\mathcal{U}_{p c}}$ (for polynomial or rational 
systems depending on the model of the phenomenon). A set $\widetilde{\mathcal{U}_{p c}}$ of admissible inputs for rational systems is defined as a subset of $\mathcal{U}_{p c}$ such that:

(i) $\forall u \in \widetilde{\mathcal{U}_{p c}} \forall t \in\left[0, T_{u}\right): u_{[0, t)} \in \widetilde{\mathcal{U}_{p c}}$,

(ii) $\forall u \in \widetilde{\mathcal{U}_{p c}} \forall \alpha \in U \exists t>0:(u)(\alpha, t) \in \widetilde{\mathcal{U}_{p c}}$,

(iii) $\forall u=\left(\alpha_{1}, t_{1}\right) \ldots\left(\alpha_{k}, t_{k}\right) \in \widetilde{\mathcal{U}_{p c}} \exists \delta>0 \forall \overline{t_{i}} \in\left[0, t_{i}+\delta\right), i=1, \ldots, k$ :

$$
\bar{u}=\left(\alpha_{1}, \overline{t_{1}}\right) \ldots\left(\alpha_{k}, \overline{t_{k}}\right) \in \widetilde{\mathcal{U}_{p c}} .
$$

A set of inputs of $\mathcal{U}_{p c}$ which satisfies the conditions (i), (ii) is called the set of admissible inputs for polynomial systems. By abusing notation, we denote it also by $\widetilde{\mathcal{U}_{p c}}$.

Because all restrictions of admissible inputs to shorter time domains are also admissible inputs, we reformulate the assumption on the form of the measurements such that we assume that the measurements are provided in the form $\left(u, y\left(T_{u}\right)\right)$ where $u \in \widetilde{\mathcal{U}_{p c}}$. A parametrized system $\Sigma(P)$ modeling a phenomenon which is characterized by the measurements $\left\{\left(u, y\left(T_{u}\right)\right) \mid u \in \widetilde{\mathcal{U}_{p c}}\right\}$ is such that $\widetilde{\mathcal{U}_{p c}} \subseteq \widetilde{\mathcal{U}_{p c}}(\Sigma(p))$ for all $p \in P$, otherwise $\Sigma(P)$ would not be a model of the phenomenon.

These assumptions make it possible to study structural and global identifiability of parametrizations of parametrized polynomial and parametrized rational systems by means of realization theory developed in [5] for polynomial and in $[26,25]$ for rational systems.

Because algebraic controllability of rational realizations is defined by using the inputs of a set $\widetilde{\mathcal{U}_{p c}}$, see [26, Definition 5.4], and because parametrized rational systems are families of rational realizations of the measurements, in the rest of the paper we mean by an algebraically controllable rational system a rational system $\Sigma=\left(X, f, h, x_{0}\right)$ realizing the measurements $\left\{\left(u, y\left(T_{u}\right)\right) \mid u \in \widetilde{\mathcal{U}_{p c}}\right\}$ such that $Z$-cl $\mathcal{R}\left(x_{0}\right)=Z$-cl $\left\{x\left(T_{u} ; x_{0}, u\right) \in X \mid u \in \widetilde{\mathcal{U}_{p c}}\right\}=X$.

Definition 4.1 Let $P \subseteq \mathbb{R}^{l}$ be a parameter set which is an irreducible real affine variety and let $\widetilde{\mathcal{U}_{p c}}$ be a set of admissible inputs for polynomial (rational) systems. Let $\Sigma(P)$ be a structured system such that $\widetilde{\mathcal{U}_{p c}} \subseteq \widetilde{\mathcal{U}_{p c}}(\Sigma(p))$ for all $p \in P$. We say that the parametrization $\mathcal{P}: P \rightarrow \Sigma(P)$ is

(i) globally identifiable if the map

$$
p \mapsto h^{p}\left(x^{p}\right)=\left\{\left(u, h^{p}\left(x^{p}\left(T_{u} ; x_{0}^{p}, u\right)\right)\right) \mid u \in \widetilde{\mathcal{U}_{p c}}\right\}
$$

is injective on $P$,

(ii) structurally identifiable if the map

$$
p \mapsto h^{p}\left(x^{p}\right)=\left\{\left(u, h^{p}\left(x^{p}\left(T_{u} ; x_{0}^{p}, u\right)\right)\right) \mid u \in \widetilde{\mathcal{U}_{p c}}\right\}
$$


is injective on $P \backslash S$ where $S$ is a variety strictly contained in $P$.

Global identifiability of a parametrization of a structured system means that the parameters of the structured system can be determined uniquely from the measurements. Structural identifiability of a parametrization provides this uniqueness only on a $Z$-dense subset of a parameter set. Obviously, a globally identifiable parametrization of a structured system is structurally identifiable.

\subsection{Structural identifiability of polynomial systems}

In this section we determine necessary and sufficient conditions for a given parametrization of a structured polynomial system to be structurally identifiable. The results are based on the realization theory for polynomial systems developed by Bartosiewicz in [5]. The realization problem as it is stated in [5] is to find for a map $\mathcal{R}: \widetilde{\mathcal{U}_{p c}} \rightarrow \mathbb{R}^{r}$ (also called a response map) a polynomial system (realization) $\Sigma=\left(X, f, h, x_{0}\right)$ such that $\mathcal{R}(u)=h\left(x\left(T_{u} ; x_{0}, u\right)\right)$ for all $u \in \widetilde{\mathcal{U}_{p c}} \subseteq \widetilde{\mathcal{U}_{p c}}(\Sigma)$. Recall that $\widetilde{\mathcal{U}_{p c}}$ is a set of admissible inputs for polynomial systems with the values in an input space $U$, and $\widetilde{\mathcal{U}_{p c}}(\Sigma)$ is the set of admissible inputs for the system $\Sigma$. Note that two polynomial systems $\Sigma_{1}$ and $\Sigma_{2}$ are realizing the same response map if $h^{1}\left(x^{1}\left(T_{u} ; x_{0}^{1}, u\right)\right)=h^{2}\left(x^{2}\left(T_{u} ; x_{0}^{2}, u\right)\right)$ for all $u \in \widetilde{\mathcal{U}_{p c}} \subseteq \widetilde{\mathcal{U}_{p c}}\left(\Sigma_{1}\right) \cap \widetilde{\mathcal{U}_{p c}}\left(\Sigma_{2}\right)$.

Theorem 4.2 [5, Theorem 4] Any two canonical polynomial realizations of the same response map are isomorphic.

Theorem 4.3 Let $P \subseteq \mathbb{R}^{l}$ be a parameter set and let $\Sigma(P)$ be a structured polynomial system with the parametrization $\mathcal{P}: P \rightarrow \Sigma(P)$. We assume that $\Sigma(P)$ is structurally canonical and we denote by $C O$ the smallest strict subvariety of $P$ such that $\Sigma(p) \in \Sigma(P)$ is canonical for all $p \in P \backslash C O$. We also assume that $\Sigma(P)$ is structurally distinguishing parameters and we denote by $D$ the smallest strict subvariety of $P$ such that $\Sigma(p)$ is distinguishing parameters for every $p \in P \backslash D$. Then the following are equivalent:

(a) the parametrization $\mathcal{P}$ is structurally identifiable,

(b) there exists a variety $S$ such that $C O \cup D \subseteq S \subsetneq P$, and such that for any $p, p^{\prime} \in P \backslash S$ an isomorphism of the systems $\Sigma(p), \Sigma\left(p^{\prime}\right) \in \Sigma(P)$ is the identity.

\section{Proof:}

$(a) \Rightarrow(b)$ Assume that the parametrization $\mathcal{P}$ is structurally identifiable. Let $G$ be a strict subvariety of $P$ such that the map $p \mapsto h^{p}\left(x^{p}\right)$ is injective on $P \backslash G$. Because $P$ is an irreducible variety and because an union of finitely 
many varieties is a variety, $C O \cup D \cup G \subsetneq P$. Let us define $S=C O \cup D \cup G$. Then $C O \cup D \subseteq S \subsetneq P$.

Let us consider $p, p^{\prime} \in P \backslash S$ and $\Sigma(p), \Sigma\left(p^{\prime}\right) \in \Sigma(P)$. Then $\Sigma(p), \Sigma\left(p^{\prime}\right)$ are canonical and such that $h^{p}\left(x^{p}\left(T_{u} ; x_{0}^{p}, u\right)\right)=h^{p^{\prime}}\left(x^{p^{\prime}}\left(T_{u} ; x_{0}^{p^{\prime}}, u\right)\right)$ for all $u \in \widetilde{\mathcal{U}_{p c}}$. Since the map $p \mapsto h^{p}\left(x^{p}\right)$ is injective on $P \backslash G$ and hence also on $P \backslash S$, the equality $h^{p}\left(x^{p}\left(T_{u} ; x_{0}^{p}, u\right)\right)=h^{p^{\prime}}\left(x^{p^{\prime}}\left(T_{u} ; x_{0}^{p^{\prime}}, u\right)\right)$ for all $u \in \widetilde{\mathcal{U}_{p c}}$ implies that $p=p^{\prime}$. Moreover, $\Sigma(P)$ is a structured system and thus the systems $\Sigma(p)$ and $\Sigma\left(p^{\prime}\right)$, where $p=p^{\prime}$, have the same structure meaning that:

(i) $\exists \phi, \psi: X^{p} \rightarrow X^{p}$ polynomial mappings such that $\phi \circ \psi=\psi \circ \phi=1_{X^{p}}$, and

(ii) $\forall \varphi \in A^{p}, \forall \alpha \in U:\left(f_{\alpha}^{p} \varphi\right) \circ \phi=f_{\alpha}^{p}(\varphi \circ \phi)$,

(iii) $h^{p}=h^{p} \circ \phi$,

(iv) $\phi$ is defined at $x_{0}^{p}$ and $\phi\left(x_{0}^{p}\right)=x_{0}^{p}$.

Consider an arbitrary isomorphism $\phi: X^{p} \rightarrow X^{p}$ satisfying the conditions above, i.e. $\phi$ is an arbitrary isomorphism of the system $\Sigma(p)$ to itself. Let $\phi^{*}: A^{p} \rightarrow A^{p}$ be an $\mathbb{R}$-algebra isomorphism defined as $\phi^{*}(\varphi)=\varphi \circ \phi$ for all $\varphi \in A^{p}$.

Because a canonical polynomial system is algebraically observable, the observation algebra $A_{o b s}(\Sigma(p))$ of the polynomial system $\Sigma(p)$ equals the algebra $A^{p}$ of all polynomials on $X^{p}$. Then the polynomials $h^{p}, f_{\alpha}^{p} h^{p}, \alpha=\left(\alpha_{1}, \ldots, \alpha_{n}\right), n \in$ $\mathbb{N}, \alpha_{i} \in U$ generate the algebra $A^{p}$. From (ii) and (iii) above, we get that

$$
\phi^{*} h^{p}=h^{p}, \text { and } \phi^{*}\left(f_{\alpha}^{p} h^{p}\right)=f_{\alpha}^{p} h^{p} \text { for } \alpha=\left(\alpha_{1}, \ldots, \alpha_{n}\right), n \in \mathbb{N}, \alpha_{i} \in U .
$$

Since the isomorphism $\phi^{*}$ maps the generators of $A^{p}$ to themselves identically, $\phi^{*}$ is the identity on $A^{p}$ and therefore the isomorphism $\phi$ is the identity on $X^{p}$.

$(b) \Rightarrow(a)$ Let $S$ be a variety such that $C O \cup D \subseteq S \subsetneq P$, and for any $p, p^{\prime} \in P \backslash S$ any isomorphism of the polynomial systems $\Sigma(p), \Sigma\left(p^{\prime}\right) \in \Sigma(P)$ is the identity.

Consider $p, p^{\prime} \in P \backslash S$ and the corresponding systems $\Sigma(p), \Sigma\left(p^{\prime}\right) \in \Sigma(P)$. Because $h^{p}\left(x^{p}\left(T_{u} ; x_{0}^{p}, u\right)\right)=h^{p^{\prime}}\left(x^{p^{\prime}}\left(T_{u} ; x_{0}^{p^{\prime}}, u\right)\right)$ for every $u \in \widehat{\mathcal{U}_{p c}}, \Sigma(p)$ and $\Sigma\left(p^{\prime}\right)$ are canonical polynomial realizations of the same response map $\mathcal{R}$, $\mathcal{R}(u)=h^{p}\left(x^{p}\left(T_{u} ; x_{0}^{p}, u\right)\right)=h^{p^{\prime}}\left(x^{p^{\prime}}\left(T_{u} ; x_{0}^{p^{\prime}}, u\right)\right)$ for $u \in \widetilde{\mathcal{U}_{p c}}$. According to Theorem 4.2, $\Sigma(p)$ and $\Sigma\left(p^{\prime}\right)$ are isomorphic. Let $\sigma: X^{p} \rightarrow X^{p^{\prime}}$ be an isomorphism of $\Sigma(p)$ and $\Sigma\left(p^{\prime}\right)$. Since $p, p^{\prime} \in P \backslash S$, (b) implies that $\sigma$ is the identity and therefore the conditions $(i)-(i v)$ of Definition 3.6, which characterize this isomorphic relation, take the form:

(i) $X^{p}=X^{p^{\prime}}$, and then also $A^{p}=A^{p^{\prime}}$,

(ii) $h^{p}=h^{p^{\prime}}$,

(iii) $\forall \varphi \in A^{p}, \forall \alpha \in U: f_{\alpha}^{p^{\prime}} \varphi=f_{\alpha}^{p} \varphi$, 
(iv) $x_{0}^{p}=x_{0}^{p^{\prime}}$.

Let $f_{\alpha, i}^{p}, h^{p}, x_{0}^{p}$ and $f_{\alpha, i}^{p^{\prime}}, h^{p^{\prime}}, x_{0}^{p^{\prime}}$ for $i=1, \ldots, n_{p}$ be written in the form of (1), (2), (3) where $\varphi_{1}, \ldots, \varphi_{n_{p}}$ are the common generators of $A^{p}$ and $A^{p^{\prime}}$ corresponding to the polynomials $X_{1}, \ldots, X_{n_{p}} \in \mathbb{R}\left[X_{1}, \ldots, X_{n_{p}}\right]$. Note that because $X^{p}=X^{p^{\prime}}$ we can assume that $n_{p}=n_{p^{\prime}}$. Then there exist polynomials $q_{i ; a_{1}, \ldots, a_{n}}^{f_{\alpha}}, q_{j ; b_{1}, \ldots, b_{n}}^{h}, q_{i}^{x_{0}}, q_{i ; a_{1}, \ldots, a_{n}}^{f^{\prime}}, q_{j ; b_{1}, \ldots, b_{n}}^{h^{\prime}}, q_{i}^{x_{0}^{\prime}} \in \mathbb{R}\left[P_{1}, \ldots, P_{l}\right], i=1, \ldots, n_{p}, j=$ $1, \ldots, r, \alpha \in U$ such that for every $\alpha \in U$ and for $i=1, \ldots, n_{p}, j=1, \ldots, r$ it holds that

$$
\begin{aligned}
f_{\alpha, i}^{p} & =\sum_{a_{1}, \ldots, a_{n_{p}} \in \mathbb{N}} q_{i ; a_{1}, \ldots, a_{n_{p}}}^{f_{\alpha}}\left(p_{1}, \ldots, p_{l}\right) \varphi_{1}^{a_{1}} \ldots \varphi_{n_{p}}^{a_{n_{p}}}, \\
h_{j}^{p} & =\sum_{b_{1}, \ldots, b_{n_{p}} \in \mathbb{N}} q_{j ; b_{1}, \ldots, b_{n_{p}}}^{h}\left(p_{1}, \ldots, p_{l}\right) \varphi_{1}^{b_{1}} \ldots \varphi_{n_{p}}^{b_{n_{p}}}, \\
x_{i}^{p}(0) & =q_{i}^{x_{0}}\left(p_{1}, \ldots, p_{l}\right),
\end{aligned}
$$

and

$$
\begin{aligned}
f_{\alpha, i}^{p^{\prime}} & =\sum_{a_{1}, \ldots, a_{n_{p}} \in \mathbb{N}} q_{i ; a_{1}, \ldots, a_{n_{p}}}^{f^{\prime}}\left(p_{1}^{\prime}, \ldots, p_{l}^{\prime}\right) \varphi_{1}^{a_{1}} \ldots \varphi_{n_{p}}^{a_{n_{p}}}, \\
h_{j}^{p^{\prime}} & =\sum_{b_{1}, \ldots, b_{n_{p}} \in \mathbb{N}} q_{j ; b_{1}, \ldots, b_{n_{p}}}^{h^{\prime}}\left(p_{1}^{\prime}, \ldots, p_{l}^{\prime}\right) \varphi_{1}^{b_{1}} \ldots \varphi_{n_{p}}^{b_{n_{p}}}, \\
x_{i}^{p^{\prime}}(0) & =q_{i}^{x_{0}^{\prime}}\left(p_{1}^{\prime}, \ldots, p_{l}^{\prime}\right) .
\end{aligned}
$$

The polynomials $q_{i ; a_{1}, \ldots, a_{n_{p}}}^{f_{\alpha}}, q_{j ; b_{1}, \ldots, b_{n_{p}}}^{h}, q_{i ; a_{1}, \ldots, a_{n_{p}}}^{f_{\alpha}^{\prime}}, q_{j ; b_{1}, \ldots, b_{n_{p}}}^{h^{\prime}} \in \mathbb{R}\left[P_{1}, \ldots, P_{l}\right]$ are non-zero polynomials only for finitely many $\left(a_{1}, \ldots, a_{n_{p}}\right),\left(b_{1}, \ldots, b_{n_{p}}\right) \in \mathbb{N}^{n_{p}}$. From (ii)-(iv) above and from the equalities (7), (8) it follows that

$$
\begin{aligned}
q_{i ; a_{1}, \ldots, a_{n_{p}}}^{f_{\alpha}}\left(p_{1}, \ldots, p_{l}\right) & =q_{i ; a_{1}, \ldots, a_{n_{p}}}^{f_{\alpha}^{\prime}}\left(p_{1}^{\prime}, \ldots, p_{l}^{\prime}\right), \\
q_{j ; b_{1}, \ldots, b_{n_{p}}}^{h}\left(p_{1}, \ldots, p_{l}\right) & =q_{j ; b_{1}, \ldots, b_{n_{p}}}^{h^{\prime}}\left(p_{1}^{\prime}, \ldots, p_{l}^{\prime}\right), \\
q_{i}^{x_{0}}\left(p_{1}, \ldots, p_{l}\right) & =q_{i}^{x_{0}^{\prime}}\left(p_{1}^{\prime}, \ldots, p_{l}^{\prime}\right) .
\end{aligned}
$$

Because $\Sigma(p), \Sigma\left(p^{\prime}\right) \in \Sigma(P)$ and $\Sigma(P)$ is a structured system, the systems $\Sigma(p)$ and $\Sigma\left(p^{\prime}\right)$ have the same structure in the sense of Definition 3.7. Therefore the relations (ii)-(iv) and consequently the equations (9) are valid even if we formally identify $p_{1}=p_{1}^{\prime}, \ldots, p_{l}=p_{l}^{\prime}$. This implies that $q_{i ; a_{1}, \ldots, a_{n_{p}}}^{f_{\alpha}}=q_{i ; a_{1}, \ldots, a_{n_{p}}}^{f^{\prime}}$, $q_{j ; b_{1}, \ldots, b_{n_{p}}}^{h}=q_{j ; b_{1}, \ldots, b_{n_{p}}}^{h^{\prime}}, q_{i}^{x_{0}}=q_{i}^{x_{0}^{\prime}}$ for $i=1, \ldots, n_{p}, j=1, \ldots, r$, and $\alpha \in U$. After substituting these relations into (9), we derive that

$$
\begin{aligned}
q_{i ; a_{1}, \ldots, a_{n_{p}}}^{f_{\alpha}}\left(p_{1}, \ldots, p_{l}\right) & =q_{i ; a_{1}, \ldots, a_{n_{p}}}^{f_{\alpha}}\left(p_{1}^{\prime}, \ldots, p_{l}^{\prime}\right), \\
q_{j ; b_{1}, \ldots, b_{n_{p}}}^{h}\left(p_{1}, \ldots, p_{l}\right) & =q_{j ; b_{1}, \ldots, b_{n_{p}}}^{h}\left(p_{1}^{\prime}, \ldots, p_{l}^{\prime}\right), \\
q_{i}^{x_{0}}\left(p_{1}, \ldots, p_{l}\right) & =q_{i}^{x_{0}}\left(p_{1}^{\prime}, \ldots, p_{l}^{\prime}\right) .
\end{aligned}
$$


Because $p, p^{\prime} \in P \backslash S$, the systems $\Sigma(p)$ and $\Sigma\left(p^{\prime}\right)$ are distinguishing parameters, i.e. $\mathbb{R}\left[\left\{q_{i ; a_{1}, \ldots, a_{n_{p}}}^{f_{\alpha}}, q_{j ; b_{1}, \ldots, b_{n_{p}}}^{h}, q_{i}^{x_{0}} \mid i=1, \ldots, n_{p}, j=1, \ldots, r, \alpha \in U\right\}\right] \cong A^{P}$. Let us denote $\mathcal{Q}=\mathbb{R}\left[\left\{q_{i ; a_{1}, \ldots, a_{n_{p}}}^{f_{\alpha}}, q_{j ; b_{1}, \ldots, b_{n_{p}}}^{h}, q_{i}^{x_{0}} \mid i=1, \ldots, n_{p}, j=1, \ldots, r, \alpha \in\right.\right.$ $U\}]$. It holds that

$$
\left(\forall q \in \mathcal{Q}: q\left(p_{1}, \ldots, p_{l}\right)=q\left(p_{1}^{\prime}, \ldots, p_{l}^{\prime}\right)\right) \Rightarrow p_{1}=p_{1}^{\prime}, \ldots, p_{l}=p_{l}^{\prime} .
$$

Since the polynomials $q_{i ; a_{1}, \ldots, a_{n_{p}}}^{f_{\alpha}}, q_{j ; b_{1}, \ldots, b_{n_{p}}}^{h}, q_{i}^{x_{0}} \in \mathcal{Q}$ generate $\mathcal{Q}$, the equalities (10) imply that $p_{1}=p_{1}^{\prime}, \ldots, p_{l}=p_{l}^{\prime}$. Therefore $p=p^{\prime}$ which proves that for the parameters of the set $P \backslash S$ the map $p \mapsto h^{p}\left(x^{p}\right)$ is injective and hence that the parametrization $\mathcal{P}$ is structurally identifiable.

Remark 4.4 The assumptions on $C O, D$ being the smallest varieties having the considered properties can be relaxed. They only provide the smallest lower bound on the varieties which can be considered as a variety $S$.

Remark 4.5 A theorem similar to Theorem 4.3 can be stated also for characterizing the global identifiability of a parametrization $\mathcal{P}$ of a structured polynomial system $\Sigma(P)$. If the systems $\Sigma(p)$ of $\Sigma(P)$ are canonical and distinguishing parameters for all $p \in P$, then the parametrization $\mathcal{P}$ is globally identifiable if and only if for every $p, p^{\prime} \in P$ an isomorphism of $\Sigma(p)$ and $\Sigma\left(p^{\prime}\right)$ is the identity.

In the following example we apply Theorem 4.3 to check structural identifiability of a parametrization of a structured polynomial system. The example provides an example of structurally canonical structured system $\Sigma(P)$ for which the parametrization $\mathcal{P}: P \rightarrow \Sigma(P)$ is not structurally identifiable.

Example 4.6 Consider a parameter set $P=\mathbb{R}^{3}$. Let $\Sigma(P)$ be a parametrized polynomial system such that every system $\Sigma(p)=\left(X^{p}, f^{p}=\left\{f_{\alpha}^{p}, \alpha \in \mathbb{R}\right\}, h^{p}, x_{0}^{p}\right)$ of $\Sigma(P)$ is given as:

$$
\begin{aligned}
X^{p} & =\mathbb{R}, \\
f_{\alpha}^{p} & =p_{1} \alpha \frac{\partial}{\partial x}, \alpha \in \mathbb{R}, \\
h^{p} & =p_{2} x^{2}, \\
x_{0}^{p} & =p_{3} .
\end{aligned}
$$

We consider the parametrization $\mathcal{P}: P=\mathbb{R}^{3} \rightarrow \Sigma(P)$. Note that the system $\Sigma(P)$ is a structured system.

The polynomials $h^{p}, f_{\alpha}^{p} h^{p}, \alpha=\left(\alpha_{1}, \ldots, \alpha_{n}\right), n \in \mathbb{N}, \alpha_{i} \in \mathbb{R}$ generate the observation algebra $A_{\text {obs }}(\Sigma(p))$. Thus $A_{\text {obs }}(\Sigma(p))=\mathbb{R}\left[p_{1} p_{2} x\right]$ and the system $\Sigma(p)$ is algebraically observable for every $p \in \mathbb{R}^{3} \backslash\left\{\left(0, p_{2}, p_{3}\right),\left(p_{1}, 0, p_{3}\right) \mid p_{1}, p_{2}, p_{3} \in \mathbb{R}\right\}$. Therefore, $\Sigma(P)$ is structurally algebraically observable. 
The reachable set of the differential equation $\dot{x}=p_{1} \alpha, x_{0}=x(0)=p_{3} \in \mathbb{R}$ is the set $\mathcal{R}\left(x_{0}\right)=\left\{p_{3}+p_{1} \alpha t \mid t \in \mathbb{R}\right\}$. Because $Z$-cl $\mathcal{R}\left(x_{0}\right)=\mathcal{R}\left(x_{0}\right)=\mathbb{R}$ for $p_{1} \neq 0$, the polynomial system $\Sigma(p)$ is algebraically controllable for $p \in \mathbb{R}^{3} \backslash$ $\left\{\left(0, p_{2}, p_{3}\right) \mid p_{2}, p_{3} \in \mathbb{R}\right\}$. Hence, $\Sigma(P)$ is structurally algebraically controllable.

Because $\Sigma(P)$ is both structurally algebraically observable and structurally algebraically controllable, it is also according to Proposition 3.10 structurally canonical. More precisely, a system $\Sigma(p) \in \Sigma(P)$ is canonical if $p \in \mathbb{R}^{3} \backslash C O$ where $C O=\left\{\left(0, p_{2}, p_{3}\right),\left(p_{1}, 0, p_{3}\right) \mid p_{1}, p_{2}, p_{3} \in \mathbb{R}\right\}$.

Because $P=\mathbb{R}^{3}$, the polynomials on $P$ are $A^{P}=\mathbb{R}\left[P_{1}, P_{2}, P_{3}\right]$. The only polynomials $q_{i ; a_{1}, \ldots, a_{n}}^{f_{\alpha}}, q_{j ; b_{1}, \ldots, b_{n}}^{h}, q_{i}^{x_{0}} \in \mathbb{R}\left[P_{1}, P_{2}, P_{3}\right]=A^{P}$ given by (1), (2), (3) for the system (11) such that $q_{i ; a_{1}, \ldots, a_{n}}^{f_{\alpha}}, q_{j ; b_{1}, \ldots, b_{n}}^{h}, q_{i}^{x_{0}} \neq 0$ (meaning that they are not zero polynomials) are

$$
q_{1 ; 0}^{f_{\alpha}}\left(p_{1}, p_{2}, p_{3}\right)=\alpha p_{1}, \alpha \in \mathbb{R}, q_{1 ; 2}^{h}\left(p_{1}, p_{2}, p_{3}\right)=p_{2}, q_{1}^{x_{0}}\left(p_{1}, p_{2}, p_{3}\right)=p_{3} .
$$

The algebra generated by $q_{1 ; 0}^{f_{\alpha}}, \alpha \in \mathbb{R}, q_{1 ; 2}^{h}$, and $q_{1}^{x_{0}}$ over $\mathbb{R}$ equals $A^{P}$. Therefore, by Definition 3.3, $\Sigma(p)$ is distinguishing parameters for every $p \in \mathbb{R}^{3} \backslash D$ where $D=\left\{\left(0, p_{2}, p_{3}\right),\left(p_{1}, 0, p_{3}\right),\left(p_{1}, p_{2}, 0\right) \mid p_{1}, p_{2}, p_{3} \in \mathbb{R}\right\}$.

Since the parametrized polynomial system $\Sigma(P)$ satisfies the assumptions of Theorem 4.3, we check the structural identifiability of the parametrization $\mathcal{P}$ by applying this theorem. Consider $p, p^{\prime} \in \mathbb{R}^{3} \backslash(C O \cup D)$. Both $\Sigma(p)$ and $\Sigma\left(p^{\prime}\right)$ are canonical and thus also isomorphic. Let $\sigma, \psi: \mathbb{R} \rightarrow \mathbb{R}$ be polynomial mappings such that $\sigma \circ \psi=\psi \circ \sigma=1_{\mathbb{R}}$ and let $\sigma$ have the properties:

(i) $p_{2} x^{2}=p_{2}^{\prime}(\sigma(x))^{2}$ for $x \in \mathbb{R}$,

(ii) $\forall \varphi \in \mathbb{R}[X], \forall \alpha \in \mathbb{R}:\left(p_{1}^{\prime} \alpha \frac{\partial}{\partial x} \varphi\right) \circ \sigma=p_{1} \alpha \frac{\partial}{\partial x}(\varphi \circ \sigma)$,

(iii) $\sigma\left(p_{3}\right)=p_{3}^{\prime}$.

Let us consider a polynomial $\varphi$ in the condition (ii) to be the identity, i.e. $\varphi(x)=x$. Since $p_{1}, p_{1}^{\prime} \neq 0$, the equality $p_{1}^{\prime} \alpha=p_{1} \alpha \frac{\partial}{\partial x} \sigma$ for all $\alpha \in \mathbb{R}$ implies that $\frac{p_{1}^{\prime}}{p_{1}}=\frac{\partial}{\partial x} \sigma$. Then the isomorphism $\sigma$ has to be of the form

$$
\sigma(x)=\frac{p_{1}^{\prime}}{p_{1}} x+c \text { for some } c \in \mathbb{R} .
$$

By substituting this form of $\sigma$ into condition (i) we derive the equality $p_{2} x^{2}=$ $p_{2}^{\prime}\left(\frac{p_{1}^{\prime 2}}{p_{1}^{2}} x^{2}+2 \frac{p_{1}^{\prime}}{p_{1}} c x+c^{2}\right)$ which implies the equations: $p_{2}=p_{2}^{\prime} \frac{p_{1}^{\prime 2}}{p_{1}^{2}}, 0=p_{2}^{\prime} \frac{p_{1}^{\prime}}{p_{1}} c$, and $0=p_{2}^{\prime} c^{2}$. Because $p_{1}, p_{1}^{\prime}, p_{2}, p_{2}^{\prime} \neq 0$, we derive that $c=0$. Therefore,

$$
\sigma(x)=\frac{p_{1}^{\prime}}{p_{1}} x
$$

From this form of $\sigma$ and from the condition (iii) it follows that $\frac{p_{1}^{\prime}}{p_{1}} p_{3}=p_{3}^{\prime}$. 
Since $p, p^{\prime} \in \mathbb{R}^{3} \backslash(C O \cup D)$ it follows that $\frac{p_{1}^{\prime}}{p_{1}}=\frac{p_{3}^{\prime}}{p_{3}}$. To get that $\sigma$ is the identity, i.e. $\sigma(x)=\frac{p_{1}^{\prime}}{p_{1}} x=x$, we restrict the parameters to be from a subset of $\mathbb{R}^{3} \backslash(C O \cup D)$ such that either $p_{3}=p_{3}^{\prime}$ or $p_{1}=p_{1}^{\prime}$. The only sets of parameters for which the parametrization $\mathcal{P}$ is globally identifiable are

$$
\begin{aligned}
& S_{\zeta}=\left\{\left(p_{1}, p_{2}, p_{3}\right) \in \mathbb{R}^{3} \backslash(C O \cup D) \mid p_{3}=\zeta\right\} \text { for } \zeta \in \mathbb{R} \backslash\{0\}, \\
& S_{\xi}=\left\{\left(p_{1}, p_{2}, p_{3}\right) \in \mathbb{R}^{3} \backslash(C O \cup D) \mid p_{1}=\xi\right\} \text { for } \xi \in \mathbb{R} \backslash\{0\} .
\end{aligned}
$$

Furthermore, because $p_{1}=p_{1}^{\prime}$ if and only if $p_{3}=p_{3}^{\prime}$, the only parameter sets for which $\mathcal{P}$ is globally identifiable are the sets $S_{\zeta} \cap S_{\xi}$ for some $\zeta, \xi \in \mathbb{R} \backslash\{0\}$. Since there does not exist a variety $V \subsetneq \mathbb{R}^{3}$ such that $\mathbb{R}^{3} \backslash V \subseteq S_{\zeta} \cap S_{\xi}$ for some $\zeta, \xi \in \mathbb{R} \backslash\{0\}$, the parametrization $\mathcal{P}: \mathbb{R}^{3} \rightarrow \Sigma(P)$ is not structurally identifiable.

Note that if we do not restrict the parameters to the parameter sets $S_{\zeta}$ or $S_{\xi}$, then the parameters $p=\left(p_{1}, p_{2}, p_{3}\right)$ and $p^{\prime}=\left(p_{1}^{\prime}, p_{2}^{\prime}, p_{3}^{\prime}\right)$ such that $p_{1}=-p_{1}^{\prime}$, $p_{2}=p_{2}^{\prime}$, and $p_{3}=-p_{3}^{\prime}$ determine two different systems $\Sigma(p), \Sigma\left(p^{\prime}\right) \in \Sigma(P)$ modeling the same phenomenon, i.e. $h^{p}\left(x^{p}\right)=h^{p^{\prime}}\left(x^{p^{\prime}}\right)$.

\subsection{Structural identifiability of rational systems}

To derive the characterization of structural identifiability of a parametrization of a structured rational system by means of realization theory we proceed in the same way as in Section 4.1 for polynomial systems. We refer to the results of realization theory for rational systems in $[26,25]$. The main result which is applied to obtain this characterization is the following:

Theorem 4.7 [25, Theorem 8] Any two canonical rational realizations of the same response map are birationally equivalent.

This theorem corresponds to Theorem 4.2 in polynomial case. The main result of this section which is a counterpart to the result stated in Theorem 4.3 is summarized in the following theorem.

Theorem 4.8 Let $P \subseteq \mathbb{R}^{l}$ be a parameter set and let $\Sigma(P)$ be a structured rational system with the parametrization $\mathcal{P}: P \rightarrow \Sigma(P)$. We assume that $\Sigma(P)$ is structurally canonical and we denote by $C O$ the smallest strict subvariety of $P$ such that $\Sigma(p) \in \Sigma(P)$ is canonical for all $p \in P \backslash C O$. We also assume that $\Sigma(P)$ is structurally distinguishing parameters and we denote by $D$ the smallest strict subvariety of $P$ such that $\Sigma(p)$ is distinguishing parameters for every $p \in P \backslash D$. Then the following are equivalent:

(a) the parametrization $\mathcal{P}$ is structurally identifiable 
(b) there exists a variety $S$ such that $C O \cup D \subseteq S \subsetneq P$, and such that for any $p, p^{\prime} \in P \backslash S$ a birational isomorphism relating the systems $\Sigma(p), \Sigma\left(p^{\prime}\right)$ is the identity.

\section{Proof:}

$(a) \Rightarrow(b)$ Let $G \subsetneq P$ be a strict subvariety of $P$ such that for $p \in P \backslash G$ the map $p \mapsto h^{p}\left(x^{p}\right)$ is injective. We define $S=C O \cup D \cup G$. It is a strict subvariety of $P$. Consider two rational systems $\Sigma(p), \Sigma\left(p^{\prime}\right) \in \Sigma(P)$ with $p, p^{\prime} \in P \backslash S$. They are both canonical and such that $h^{p}\left(x^{p}\left(T_{u} ; x_{0}^{p}, u\right)\right)=h^{p^{\prime}}\left(x^{p^{\prime}}\left(T_{u} ; x_{0}^{p^{\prime}}, u\right)\right)$ for all $u \in \widetilde{\mathcal{U}_{p c}}$. By Theorem 4.7, $\Sigma(p)$ and $\Sigma\left(p^{\prime}\right)$ are birationally equivalent. Further, according to $(a), p=p^{\prime}$. From Definition 3.6 it follows that

(i) there exist rational mappings $\phi: X^{p} \rightarrow X^{p}, \psi: X^{p} \rightarrow X^{p}$ such that $\phi \circ \psi=1_{X^{p}}$ and $\psi \circ \phi=1_{X^{p}}$ (these mappings do not have to be defined everywhere, only on $Z$-dense subsets of $X^{p}$ ),

(ii) $h^{p} \circ \phi=h^{p}$,

(iii) $\forall \varphi \in Q^{p}, \forall \alpha \in U: f_{\alpha}^{p}(\varphi \circ \phi)=\left(f_{\alpha}^{p} \varphi\right) \circ \phi$,

(iv) $\phi$ is defined at $x_{0}^{p}$ and $\phi\left(x_{0}^{p}\right)=x_{0}^{p}$.

As $\Sigma(p)$ is canonical and thus rationally observable, $Q_{o b s}(\Sigma(p))=Q^{p}$. Because the field $Q_{o b s}(\Sigma(p))$ is generated by the rational functions $h^{p}, f_{\alpha}^{p}, \alpha=$ $\left(\alpha_{1}, \ldots, \alpha_{n}\right), n \in \mathbb{N}, \alpha_{i} \in U$, and because from (ii) and (iii) above $h^{p} \circ \phi=$ $h^{p}, f_{\alpha}^{p} h^{p}=f_{\alpha}^{p}\left(h^{p} \circ \phi\right)=\left(f_{\alpha}^{p} h^{p}\right) \circ \phi$ for $\alpha=\left(\alpha_{1}, \ldots, \alpha_{n}\right), n \in \mathbb{N}, \alpha_{i} \in U$, we get that

$$
\forall \varphi \in Q_{o b s}(\Sigma(p))=Q^{p}: \varphi=\varphi \circ \phi \text { on } X^{p} .
$$

Especially for $\varphi \in Q^{p}$ defined as $\varphi(x)=x$ it means that $x=\varphi(x)=\varphi(\phi(x))=$ $\phi(x)$. Therefore, a birational isomorphism $\phi: X^{p} \rightarrow X^{p}$ relating the systems $\Sigma(p), \Sigma\left(p^{\prime}\right) \in \Sigma(P)$ for $p, p^{\prime} \in P \backslash S$ is the identity.

Note that the property of distinguishing parameters for the systems $\Sigma(p)$, $p \in P \backslash S$ is not needed in the proof of this implication. If the statement of (b) assumed for a variety $S$ only the condition $C O \subseteq S \subsetneq P$, then it would be sufficient to define a variety $S$ as $S=C O \cup G$ to prove the implication $(a) \Rightarrow(b)$.

$(b) \Rightarrow(a)$ Let $p, p^{\prime} \in P \backslash S$. Then $\Sigma(p), \Sigma\left(p^{\prime}\right) \in \Sigma(P)$ are two rational systems which are canonical, distinguishing parameters, and such that for all $u \in \widetilde{\mathcal{U}}_{p c}$ $h^{p}\left(x^{p}\left(T_{u} ; x_{0}^{p}, u\right)\right)=h^{p^{\prime}}\left(x^{p^{\prime}}\left(T_{u} ; x_{0}^{p^{\prime}}, u\right)\right)$. From Theorem 4.7, the systems $\Sigma(p)$ and $\Sigma\left(p^{\prime}\right)$ are birationally equivalent. Because $p, p^{\prime} \in P \backslash S$, we get from $(b)$ that a birational isomorphism $\phi$ relating $\Sigma(p)$ and $\Sigma\left(p^{\prime}\right)$ is the identity, i.e. the systems $\Sigma(p), \Sigma\left(p^{\prime}\right)$ are related in the following way:

(i) $X^{p} \cap X^{p^{\prime}}$ is a $Z$-dense subset of both $X^{p}$ and $X^{p^{\prime}}$,

(ii) $h^{p^{\prime}}=h^{p}$,

(iii) $\forall \varphi \in Q^{p^{\prime}}, \forall \alpha \in U: f_{\alpha}^{p} \varphi=f_{\alpha}^{p^{\prime}} \varphi$, 
(iv) $x_{0}^{p}=x_{0}^{p^{\prime}}$.

Since both $\Sigma(p)$ and $\Sigma\left(p^{\prime}\right)$ are algebraically controllable, $Z$-cl $\mathcal{R}\left(x_{0}^{p}\right)=X^{p}$ and $Z$-cl $\mathcal{R}\left(x_{0}^{p^{\prime}}\right)=X^{p^{\prime}}$. Moreover, by (iii) and (iv), the trajectories determining the reachable sets $\mathcal{R}\left(x_{0}^{p}\right)$ and $\mathcal{R}\left(x_{0}^{p^{\prime}}\right)$ coincide. Therefore $Z$-cl $\mathcal{R}\left(x_{0}^{p}\right)=Z$-cl $\mathcal{R}\left(x_{0}^{p^{\prime}}\right)$, thus $X^{p}=X^{p^{\prime}}$, and consequently $Q^{p}=Q^{p^{\prime}}$.

By following the steps of the proof of Theorem 4.3 and by considering $f_{\alpha, i}^{p}, h^{p}, x_{0}^{p}$ and $f_{\alpha, i}^{p^{\prime}}, h^{p^{\prime}}, x_{0}^{p^{\prime}}$ for $i=1, \ldots, n_{p}=n_{p^{\prime}}$ to be written in the form of $(4),(5)$, (6), we derive that $p=p^{\prime}$. Hence, the parametrization $\mathcal{P}: P \rightarrow \Sigma(P)$ is structurally identifiable.

As in the polynomial case, the assumptions on $C O, D$ being the smallest varieties having the considered properties can be relaxed since their union only provides the lower bound on a variety $S$.

Remark 4.9 In the same way as we derived the characterization of global identifiability for a parametrization of a structured polynomial system from Theorem 4.3, see Remark 4.5, we can derive the characterization of global identifiability for a parametrization of a structured rational system by adjusting the theorem providing the characterization of its structural identifiability.

Consider a structured rational system $\Sigma(P)$ such that $\Sigma(p)$ is canonical and distinguishing parameters for all $p \in P \backslash W$ where $W$ is a set of such parameters $p^{W}$ from $P$ for which $\Sigma\left(p^{W}\right)$ is not well-defined. The parametrization $\mathcal{P}: P \rightarrow$ $\Sigma(P)$ is globally identifiable if and only if for every $p, p^{\prime} \in P \backslash W$ a birational isomorphism for $\Sigma(p)$ and $\Sigma\left(p^{\prime}\right)$ is the identity.

Remark 4.10 Note the similarity of Theorem 4.3 and Theorem 4.8 with [34, Theorem 2.9] which treats the structural identifiability of parametrization from the Markov and initial parameters for linear systems.

Example 4.11 In this example we consider the one-compartment model with nonlinear Michaelis-Menten elimination studied in [22, Example 5] and in [9].

Let $P=\mathbb{R}^{3}$ be the parameter set of a structured rational system $\Sigma(P)$ such that the systems $\Sigma(p)=\left(X^{p}, f^{p}, h^{p}, x_{0}^{p}\right) \in \Sigma(P)$ are given as:

$$
\begin{aligned}
X^{p} & =\mathbb{R}, \\
f_{\alpha}^{p} & =\left(-\frac{p_{1} x}{p_{3}+x}-p_{2} x+\alpha\right) \frac{\partial}{\partial x}, \alpha \in \mathbb{R}, \\
h^{p} & =x, \\
x^{p}(0) & =a, a \in \mathbb{R} \text { is known. }
\end{aligned}
$$


The system $\Sigma(p)$ is algebraically observable and algebraically controllable for $p \in P \backslash\left\{p=\left(p_{1}, p_{2}, p_{3}\right) \in P \mid p_{3}=-a\right\}$. Hence, the set $\Sigma(P)$ is structurally canonical.

Because $f_{\alpha}^{p}=\left(-\frac{p_{1} x}{p_{3}+x}-p_{2} x+\alpha\right) \frac{\partial}{\partial x}=\frac{\alpha p_{3}+\left(\alpha-p_{1}-p_{2} p_{3}\right) x-p_{2} x^{2}}{p_{3}+x} \frac{\partial}{\partial x}$ and $h^{p}=\frac{x}{1}$, it follows that if we write $f_{\alpha, 1}^{p}$ and $h^{p}$ in the form of (4) and (5), respectively, then for $p=\left(p_{1}, p_{2}, p_{3}\right) \in P$ the polynomials $q_{1,1 ; 0}^{f_{\alpha}}(p)=\alpha p_{3}, q_{1,1 ; 1}^{f_{\alpha}}(p)=\alpha-p_{1}-p_{2} p_{3}$, $q_{1,1 ; 2}^{f_{\alpha}}(p)=-p_{2}, q_{2,1 ; 0}^{f_{\alpha}}(p)=p_{3}, q_{2,1 ; 1}^{f_{\alpha}}(p)=1, q_{1,1 ; 1}^{h}(p)=1$, and $q_{2,1 ; 0}^{h}(p)=1$ are the only non-zero elements of $\mathbb{R}\left(P_{1}, P_{2}, P_{3}\right)$. Since $\mathbb{R}\left(q_{1,1 ; 1}^{f_{\alpha}}, q_{1,1 ; 2}^{f_{\alpha}}, q_{2,1 ; 0}^{f_{\alpha}}\right)=$ $\mathbb{R}\left(P_{1}, P_{2}, P_{3}\right)=Q^{P}$, it follows from Definition 3.4 that the system $\Sigma(p)$ is distinguishing parameters for $p \in P \backslash\left\{p=\left(p_{1}, p_{2}, p_{3}\right) \in P \mid\left(p_{2} p_{3}\right)^{2}+\left(p_{1}+\right.\right.$ $\left.\left.p_{2} p_{3}\right)^{2}=0\right\}$.

Let us consider $p, p^{\prime} \in P \backslash\left\{p=\left(p_{1}, p_{2}, p_{3}\right) \in P \mid\left(p_{3}+a\right)^{2}+\left(p_{2} p_{3}\right)^{2}+\left(p_{1}+p_{2} p_{3}\right)^{2}=\right.$ $0\}$. From Definition 3.6 and Theorem 4.7 there exists a birational isomorphism $\phi: X^{p} \rightarrow X^{p^{\prime}}$ which satisfies for example that $h^{p^{\prime}} \phi=h^{p}$. Because $h^{p}(x)=$ $h^{p^{\prime}}(x)=x$, it follows that $\phi(x)=h^{p^{\prime}}(\phi(x))=h^{p}(x)=x$. Thus, $\phi$ is the identity. By Theorem 4.8 we conclude that the parametrization $\mathcal{P}: P \rightarrow \Sigma(P)$ is structurally identifiable.

Because $\Sigma(p)$ is not distinguishing parameters for all $p \in P \backslash W$ where $W$ is a set of all parameters $p^{W} \in P$ for which $\Sigma\left(p^{W}\right)$ is not well-defined, we cannot decide whether the parametrization $\mathcal{P}$ is globally identifiable only by applying the theorem of Remark 4.9.

\section{Examples}

In this section we apply the results we derived to study the identifiability properties of the systems modeling different biological phenomena.

Example 5.1 Consider a chain of two enzyme-catalyzed irreversible reactions represented by the following diagram:

$$
\longrightarrow x_{1} \frac{E_{1}}{\frac{p_{1} x_{1}}{p_{2}+x_{1}}} \longrightarrow x_{2} \underset{\frac{E_{2}}{p_{4}+x_{2}}}{\longrightarrow}
$$

We assume that the inputs $u$ are piecewise constant functions. The corresponding rates $\frac{p_{1} x_{1}}{p_{2}+x_{1}}$ and $\frac{p_{3} x_{2}}{p_{4}+x_{2}}$ of the reactions catalyzed by the enzymes $E_{1}$ and $E_{2}$ are modeled by Michaelis-Menten kinetics. Let $U$ be the set of all real values of admissible inputs $u$ and let $P=\left\{p=\left(p_{1}, p_{2}, p_{3}, p_{4}\right) \in \mathbb{R}^{4}\right\}$ be the parameter set. The parametrized system $\Sigma(P)$ modeling this chain of the reactions 
consists of rational systems $\Sigma(p), p \in P$ of the following form:

$$
\begin{aligned}
X^{p} & =\mathbb{R}^{2}, \\
f_{\alpha}^{p} & =\left(-\frac{p_{1} x_{1}}{p_{2}+x_{1}}+\alpha\right) \frac{\partial}{\partial x_{1}}+\left(\frac{p_{1} x_{1}}{p_{2}+x_{1}}-\frac{p_{3} x_{2}}{p_{4}+x_{2}}\right) \frac{\partial}{\partial x_{2}}, \alpha \in U, \\
h^{p}\left(x_{1}, x_{2}\right) & =\left(h_{1}^{p}\left(x_{1}, x_{2}\right), h_{2}^{p}\left(x_{1}, x_{2}\right)\right)=\left(\frac{p_{1} x_{1}}{p_{2}+x_{1}}, \frac{p_{3} x_{2}}{p_{4}+x_{2}}\right), \\
x_{1}^{p}(0) & =1, \quad x_{2}^{p}(0)=1 .
\end{aligned}
$$

Consider an arbitrary $p \in P$. We derive the conditions on $p$ which have to be satisfied for $\Sigma(p)$ to be algebraically observable. First of all, $h_{1}^{p}=\frac{p_{1} x_{1}}{p_{2}+x_{1}}, h_{2}^{p}=$ $\frac{p_{3} x_{2}}{p_{4}+x_{2}} \in Q_{o b s}(\Sigma)$. If $p_{1}, p_{3} \neq 0$, then also $\frac{x_{1}}{p_{2}+x_{1}}, \frac{x_{2}}{p_{4}+x_{2}} \in Q_{o b s}(\Sigma)$. If $p_{4} \neq 0$ then $f_{\alpha}^{p}\left(\frac{x_{2}}{p_{4}+x_{2}}\right)=\frac{p_{4}}{\left(p_{4}+x_{2}\right)^{2}}\left(h_{1}^{p}+h_{2}^{p}\right) \in Q_{o b s}(\Sigma)$ implies that $\left(p_{4}+x_{2}\right)^{2} \in Q_{o b s}(\Sigma)$. By multiplying the elements $\left(p_{4}+x_{2}\right)^{2}$ and $\frac{x_{2}^{2}}{\left(p_{4}+x_{2}\right)^{2}}$ of $Q_{o b s}(\Sigma)$, we derive that $x_{2}^{2} \in Q_{o b s}(\Sigma)$. Therefore, if $p_{4} \neq 0$, the element $\frac{\left(p_{4}+x_{2}\right)^{2}-x_{2}^{2}-p_{4}^{2}}{2 p_{4}}=x_{2}$ belongs to $Q_{\text {obs }}(\Sigma)$. By considering $f_{\alpha}^{p}\left(\frac{x_{1}}{p_{2}+x_{1}}\right) \in Q_{\text {obs }}(\Sigma)$ we derive in an analogous way that if $p_{2} \neq 0$ then $x_{1} \in Q_{o b s}(\Sigma)$. Thus, the systems $\Sigma(p)$ for $p \in P \backslash$ $\left\{p=\left(p_{1}, p_{2}, p_{3}, p_{4}\right) \in \mathbb{R}^{4} \mid p_{1} p_{2} p_{3} p_{4}=0\right\}$ are algebraically observable and the parametrized system $\Sigma(P)$ is structurally algebraically observable.

Note that to check algebraic controllability of a system $\Sigma(p) \in \Sigma(P)$ it is sufficient to prove that its reachable set from the initial state contains a non-empty open set. Then the Zariski closure of the reachable set equals $\mathbb{R}^{2}$ implying that $\Sigma(p)$ is algebraically controllable. Because the system $\Sigma(p)$ is a smooth affine nonlinear control system, we can apply for example [23, Theorem 3.9] or [18, Theorem 2.2.4] to prove that the reachable set of $\Sigma(p)$ contains a non-empty open set in $\mathbb{R}^{2}$. By using the terminology of [23], it is sufficient to prove that the accessibility distribution $C$ at $x_{0}^{p}$ has a dimension 2 . Since the accessibility algebra is spanned by the vector fields $f=-\frac{p_{1} x_{1}}{p_{2}+x_{1}} \frac{\partial}{\partial x_{1}}+\left(\frac{p_{1} x_{1}}{p_{2}+x_{1}}-\frac{p_{3} x_{2}}{p_{4}+x_{2}}\right) \frac{\partial}{\partial x_{2}}, g=\frac{\partial}{\partial x_{1}}$, and their Lie brackets, it follows that for $C\left(x_{0}^{p}\right)=C\left(\left(x_{1}^{p}(0), x_{2}^{p}(0)\right)\right)=C((1,1))$ we get that

$$
\operatorname{dim} C\left(x_{0}^{p}\right)=\operatorname{dim}\left(\begin{array}{cc}
\frac{-p_{1}}{p_{2}+1} & 1 \\
\frac{p_{1}}{p_{2}+1}-\frac{p_{3}}{p_{4}+1} & 0
\end{array}\right) .
$$

If $p_{2}, p_{4} \neq-1$ and $p_{1}\left(p_{4}+1\right)-p_{3}\left(p_{2}+1\right) \neq 0$ then $\operatorname{dim} C\left(x_{0}^{p}\right)=2$ and consequently the system $\Sigma(p)$ is algebraically controllable from the initial state $\left(x_{1}^{p}(0), x_{2}^{p}(0)\right)$. Finally, the systems $\Sigma(p)$ for $p \in P \backslash\left\{\left(p_{1}, p_{2}, p_{3}, p_{4}\right) \in \mathbb{R}^{4} \mid\left(p_{2}+\right.\right.$ $\left.1)^{2}+\left(p_{4}+1\right)^{2}+\left(p_{1}\left(p_{4}+1\right)-p_{3}\left(p_{2}+1\right)\right)^{2}=0\right\}$ are algebraically controllable and thus the parametrized system $\Sigma(P)$ is structurally algebraically controllable.

By Proposition 3.10, the set $\Sigma(P)$ is structurally canonical.

The systems $\Sigma(p)$ with $p \in P \backslash\left\{\left(p_{1}, \ldots, p_{4}\right) \in P \mid p_{1} p_{2} p_{3} p_{4}=0\right\}$ are distinguishing parameters. This can be checked as follows. Consider the components $h_{1}^{p}, h_{2}^{p}$ 
of $h^{p}$. From (5) we derive that $q_{1,1 ; 1,0}^{h^{p}}\left(p_{1}, \ldots, p_{4}\right)=p_{1}, q_{2,1 ; 0,0}^{h^{p}}\left(p_{1}, \ldots, p_{4}\right)=$ $p_{2}, q_{1,2 ; 0,1}^{h^{p}}\left(p_{1}, \ldots, p_{4}\right)=p_{3}$, and $q_{2,2 ; 0,0}^{h^{p}}\left(p_{1}, \ldots, p_{4}\right)=p_{4}$. This implies that $\mathbb{R}\left(q_{1,1 ; 1,0}^{h^{p}}, q_{2,1 ; 0,0}^{h^{p}}, q_{1,2 ; 0,1}^{h^{p}}, q_{2,2 ; 0,0}^{h^{p}}\right)=\mathbb{R}\left(P_{1}, \ldots, P_{4}\right)$ if $p \in P \backslash\left\{\left(p_{1}, \ldots, p_{4}\right) \in\right.$ $\left.P \mid p_{1} p_{2} p_{3} p_{4}=0\right\}$. Because $P=\mathbb{R}^{4}$ and thus $Q^{P}=\mathbb{R}\left(P_{1}, \ldots, P_{4}\right)$, the system $\Sigma(P)$ is structurally distinguishing parameters.

Consider two parameters $p, p^{\prime} \in P \backslash\left\{\left(p_{1}, p_{2}, p_{3}, p_{4}\right) \in \mathbb{R}^{4} \mid\left(p_{1} p_{2} p_{3} p_{4}\right)^{2}+\left(p_{2}+\right.\right.$ $\left.1)^{2}+\left(p_{4}+1\right)^{2}+\left(p_{1}\left(p_{4}+1\right)-p_{3}\left(p_{2}+1\right)\right)^{2}=0\right\}$. Then both systems $\Sigma(p)$ and $\Sigma\left(p^{\prime}\right)$ are canonical and such that $h^{p}\left(x^{p}\left(T_{u} ; x_{0}^{p}, u\right)\right)=h^{p^{\prime}}\left(x^{p^{\prime}}\left(T_{u} ; x_{0}^{p^{\prime}}, u\right)\right)$ for all $u \in \widetilde{\mathcal{U}_{p c}}$. Therefore $\Sigma(p)$ and $\Sigma\left(p^{\prime}\right)$ are birationally equivalent according to Theorem 4.7. There exists a birational isomorphism $\phi=\left(\phi_{1}, \phi_{2}\right): \mathbb{R}^{2} \rightarrow \mathbb{R}^{2}$ such that:

(i) $h^{p^{\prime}} \phi=h^{p}$,

(ii) $\forall \varphi \in Q^{p^{\prime}}, \forall \alpha \in U: f_{\alpha}^{p}(\varphi \circ \phi)=\left(f_{\alpha}^{p^{\prime}} \varphi\right) \circ \phi$,

(iii) $\phi\left(x_{0}^{p}\right)=x_{0}^{p^{\prime}}$.

By substituting the explicit forms of $h^{p}$ and $h^{p^{\prime}}$ into (i) we derive the equation

$$
\frac{p_{1}^{\prime} \phi_{1}\left(x_{1}, x_{2}\right)}{p_{2}^{\prime}+\phi_{1}\left(x_{1}, x_{2}\right)}=\frac{p_{1} x_{1}}{p_{2}+x_{1}}
$$

and the equation

$$
\frac{p_{3}^{\prime} \phi_{2}\left(x_{1}, x_{2}\right)}{p_{4}^{\prime}+\phi_{2}\left(x_{1}, x_{2}\right)}=\frac{p_{3} x_{2}}{p_{4}+x_{2}}
$$

Let us consider the polynomial $\varphi\left(x_{1}, x_{2}\right)=x_{1}$ in the condition (ii) above. Then for every $\alpha \in U$ it holds that $\frac{-p_{1}^{\prime} \phi_{1}\left(x_{1}, x_{2}\right)}{p_{2}^{\prime}+\phi_{1}\left(x_{1}, x_{2}\right)}+\alpha=\left(\frac{-p_{1} x_{1}}{p_{2}+x_{1}}+\alpha\right) \frac{\partial \phi_{1}\left(x_{1}, x_{2}\right)}{\partial x_{1}}+$ $\left(\frac{p_{1} x_{1}}{p_{2}+x_{1}}-\frac{p_{3} x_{2}}{p_{4}+x_{2}}\right) \frac{\partial \phi_{1}\left(x_{1}, x_{2}\right)}{\partial x_{2}}$. By substituting (12) into this equation we obtain the equation:

$\left(\frac{p_{1} x_{1}}{p_{2}+x_{1}}-\alpha\right) \frac{\partial \phi_{1}\left(x_{1}, x_{2}\right)}{\partial x_{1}}+\left(\frac{p_{3} x_{2}}{p_{4}+x_{2}}-\frac{p_{1} x_{1}}{p_{2}+x_{1}}\right) \frac{\partial \phi_{1}\left(x_{1}, x_{2}\right)}{\partial x_{2}}=\frac{p_{1} x_{1}}{p_{2}+x_{1}}-\alpha$.

We solve this linear partial differential equation by the method of characteristics, see for example [12]. The equations for the characteristic curves are:

$$
\begin{aligned}
& \frac{d \xi}{d s}=\frac{p_{1} x_{1}}{p_{2}+x_{1}}-\alpha=\frac{p_{1} \xi(s)}{p_{2}+\xi(s)}-\alpha, \\
& \frac{d \eta}{d s}=\frac{p_{3} x_{2}}{p_{4}+x_{2}}-\frac{p_{1} x_{1}}{p_{2}+x_{1}}=\frac{p_{3} \eta(s)}{p_{4}+\eta(s)}-\frac{p_{1} \xi(s)}{p_{2}+\xi(s)} .
\end{aligned}
$$

The restriction $\phi_{1, \gamma}(s)=\phi_{1}(\xi(s), \eta(s))$ of $\phi_{1}\left(x_{1}, x_{2}\right)$ to $\gamma=\left\{\left(x_{1}, x_{2}\right) \in \mathbb{R}^{2} \mid x_{1}=\right.$ $\left.\xi(s), x_{2}=\eta(s), \xi, \eta \in \mathcal{C}^{1}(I, \mathbb{R})\right\}$ is given by the equation $\frac{d \phi_{1, \gamma}}{d s}=\frac{d \phi_{1}}{d x_{1}} \frac{d \xi}{d s}+\frac{d \phi_{1}}{d x_{2}} \frac{d \eta}{d s}$. 
From (14) it follows that

$$
\frac{d \phi_{1, \gamma}}{d s}=\frac{p_{1} \xi(s)}{p_{2}+\xi(s)}-\alpha=\frac{d \xi}{d s}
$$

and consequently $\phi_{1}(\xi(s), \eta(s))=\xi(s)+c$ where $c \in \mathbb{R}$ is a constant. Then $\phi_{1}\left(x_{1}, x_{2}\right)=x_{1}+c$ and because $\phi_{1}\left(x_{0}^{p}\right)=\phi_{1}\left(x_{1}^{p}(0), x_{2}^{p}(0)\right)=x_{1}^{p}(0)$ according to the condition (iii) above, we get that

$$
\phi_{1}\left(x_{1}, x_{2}\right)=x_{1}
$$

By considering the polynomial $\varphi\left(x_{1}, x_{2}\right)=x_{2}$ in the condition (ii) above and by substituting (13) into the derived relation, we obtain the following equation valid for all $\alpha \in U$ :

$$
\left(\frac{p_{1} x_{1}}{p_{2}+x_{1}}-\alpha\right) \frac{\partial \phi_{2}}{\partial x_{1}}+\left(\frac{p_{3} x_{2}}{p_{4}+x_{2}}-\frac{p_{1} x_{1}}{p_{2}+x_{1}}\right) \frac{\partial \phi_{2}}{\partial x_{2}}=\frac{p_{3} x_{2}}{p_{4}+x_{2}}-\frac{p_{1} x_{1}}{p_{2}+x_{1}} .
$$

Again, by the method of characteristics, we solve this linear partial differential equation. We get that $\phi_{2}\left(x_{1}, x_{2}\right)=x_{2}+c$ for $c \in \mathbb{R}$. Because $\phi_{2}\left(x_{0}^{p}\right)=$ $\phi_{2}\left(x_{1}^{p}(0), x_{2}^{p}(0)\right)=x_{2}^{p}(0)$ according to the condition (iii),

$$
\phi_{2}\left(x_{1}, x_{2}\right)=x_{2}
$$

Finally, from (15) and (16), a map $\phi$ is the identity and hence, from Theorem 4.8, the parametrization $\mathcal{P}$ is structurally identifiable.

For a comparison of the method given by Theorem 4.8 and the differential algebraic methods used in [22] for determining structural and global identifiability of the parametrizations of structured rational systems, we consider the following example of a two-compartment model with Michaelis-Menten elimination kinetics studied in [22, Section 7].

Example 5.2 Let $P=\mathbb{R}^{4}$ be the parameter set of a structured rational system $\Sigma(P)$. The rational systems $\Sigma(p)=\left(X^{p}, f^{p}, h^{p}, x_{0}^{p}=\left(x_{1}^{p}(0), x_{2}^{p}(0)\right)\right) \in \Sigma(P)$ are given as:

$$
\begin{aligned}
& X^{p} \subseteq \mathbb{R}^{2} \\
& f^{p}=\left(-p_{1} x_{1}+p_{2} x_{2}\right) \frac{\partial}{\partial x_{1}}+\left(p_{1} x_{1}-p_{2} x_{2}-\frac{p_{3} x_{2}}{p_{4}+x_{2}}\right) \frac{\partial}{\partial x_{2}}, \\
& h^{p}=x_{1} \\
& x_{1}^{p}(0)=a, a \in \mathbb{R}, x_{2}^{p}(0)=0 .
\end{aligned}
$$

For the parameters $p \in P \backslash\left\{p=\left(p_{1}, p_{2}, p_{3}, p_{4}\right) \in P \mid p_{2}=0\right\}$ the system $\Sigma(p)$ is rationally observable. 
The system $\Sigma(p)$ rewritten in the state-space form is given as

$$
\begin{aligned}
& \dot{x}_{1}=-p_{1} x_{1}+p_{2} x_{2}, \\
& \dot{x}_{2}=p_{1} x_{1}-p_{2} x_{2}-\frac{p_{3} x_{2}}{p_{4}+x_{2}}, \\
& x_{1}(0)=a \in \mathbb{R}, x_{2}(0)=0 .
\end{aligned}
$$

If $p_{3} \neq 0$, it follows that $\left(p_{4}+x_{2}\right) \dot{x}_{1}+\left(p_{4}+x_{2}\right) \dot{x}_{2}+p_{3} x_{2}=0$. Since there does not exist a polynomial $M\left(x_{1}, x_{2}\right) \in \mathbb{R}\left[x_{1}, x_{2}\right]$ such that $\frac{d}{d t} M\left(x_{1}, x_{2}\right)=$ $\left(p_{4}+x_{2}\right) \dot{x}_{1}+\left(p_{4}+x_{2}\right) \dot{x}_{2}+p_{3} x_{2}$ if we consider $x_{1}, x_{2}$ being time-dependent, the reachable set is not an algebraic variety. According to [1, Theorem 2.3], there exists a solution of (17) defined on a non-empty time interval. Because this solution cannot be described in the state space as an algebraic curve or as a finite set of points (otherwise the reachable set would be an algebraic variety), it implies that the smallest variety containing the reachable set is the whole state space. Therefore, the system $\Sigma(p)$ is algebraically controllable from the initial state $\left(x_{1}^{p}(0)=a \in \mathbb{R}, x_{2}^{p}(0)=0\right)$ for the parameters $p=\left(p_{1}, p_{2}, p_{3}, p_{4}\right) \in P$ such that $p_{3} \neq 0$.

Because the rational system $\Sigma(p)$ is rationally observable and algebraically controllable for $p \in P \backslash\left\{p=\left(p_{1}, p_{2}, p_{3}, p_{4}\right) \in P \mid p_{2} p_{3}=0\right\}$, the parametrized system $\Sigma(P)$ is structurally canonical.

Consider the vector field $f^{p}$ of a system $\Sigma(p) \in \Sigma(P)$. If we rewrite $f_{1}^{p}$ and $f_{2}^{p}$ in the form of (4) we derive that $q_{1,1 ; 1,0}^{f}\left(p_{1}, \ldots, p_{4}\right)=-p_{1}, q_{1,1 ; 0,1}^{f}\left(p_{1}, \ldots, p_{4}\right)=$ $p_{2}, q_{1,2 ; 0,1}^{f}\left(p_{1}, \ldots, p_{4}\right)=-p_{2} p_{4}-p_{3}, q_{2,2 ; 0,0}^{f}\left(p_{1}, \ldots, p_{4}\right)=p_{4}$, and consequently that $\mathbb{R}\left(q_{1,1 ; 1,0}^{f}, q_{1,1 ; 0,1}^{f}, q_{1,2 ; 0,1}^{f}, q_{2,2 ; 0,0}^{f}\right)=\mathbb{R}\left(P_{1}, P_{2}, P_{3}, P_{4}\right)=Q^{P}$ for the parameters $p \in P \backslash\left\{p=\left(p_{1}, p_{2}, p_{3}, p_{4}\right) \in P \mid p_{1} p_{2} p_{3} p_{4}=0\right\}$. Therefore $\Sigma(P)$ is structurally distinguishing parameters.

Consider two systems $\Sigma(p), \Sigma\left(p^{\prime}\right) \in \Sigma(P)$ with $p, p^{\prime} \in P \backslash\left\{p=\left(p_{1}, p_{2}, p_{3}, p_{4}\right) \in\right.$ $\left.P \mid p_{1} p_{2} p_{3} p_{4}=0\right\}$. Then $\Sigma(p)$ and $\Sigma\left(p^{\prime}\right)$ are canonical and distinguishing parameters. Further it holds that $h^{p}\left(x^{p}\right)=h^{p^{\prime}}\left(x^{p^{\prime}}\right)$. From Theorem 4.7, the systems $\Sigma(p), \Sigma\left(p^{\prime}\right)$ are birationally equivalent and it holds that

(i) there exist rational mappings $\phi, \psi$ such that $\phi: X^{p} \rightarrow X^{p^{\prime}}, \psi: X^{p^{\prime}} \rightarrow X^{p}$, and $\phi \circ \psi=1_{X^{p}}, \psi \circ \phi=1_{X^{p^{\prime}}}$; moreover, we derive that $\phi\left(x_{1}, x_{2}\right)=$ $\left(\phi_{1}\left(x_{1}, x_{2}\right), \phi_{2}\left(x_{1}, x_{2}\right)\right)$ for $x_{1}, x_{2} \in X^{p}$ and that, for $x_{1}, x_{2} \in X^{p^{\prime}}, \psi\left(x_{1}, x_{2}\right)=$ $\left(\psi_{1}\left(x_{1}, x_{2}\right), \psi_{2}\left(x_{1}, x_{2}\right)\right)$,

(ii) $\phi_{1}\left(x_{1}, x_{2}\right)=h^{p^{\prime}}\left(\phi_{1}\left(x_{1}, x_{2}\right), \phi_{2}\left(x_{1}, x_{2}\right)\right)=h^{p^{\prime}}\left(\phi\left(x_{1}, x_{2}\right)\right)=h^{p}\left(x_{1}, x_{2}\right)=x_{1}$,

(iii) $\forall \varphi \in Q_{p^{\prime}}: f^{p}(\varphi \circ \phi)=\left(f^{p^{\prime}} \varphi\right) \circ \phi$,

(iv) because the initial state is independent of the parameters, we have that $x_{1}^{p}(0)=x_{1}^{p^{\prime}}(0)=a \in \mathbb{R}, x_{2}^{p}(0)=x_{2}^{p^{\prime}}(0)=0$ and therefore $\phi\left(x^{p}(0)\right)=$ $x^{p^{\prime}}(0)=x^{p}(0)$.

By considering the polynomial $\varphi\left(x_{1}, x_{2}\right)=x_{1}$ in the condition (iii) and by 
applying the relation $\phi_{1}\left(x_{1}, x_{2}\right)=x_{1}$ from (ii) to it, we derive the equality $-p_{1} x_{1}+p_{2} x_{2}=-p_{1}^{\prime} x_{1}+p_{2}^{\prime} \phi_{2}\left(x_{1}, x_{2}\right)$. Because $p_{2}^{\prime} \neq 0$ it follows that

$$
\phi_{2}\left(x_{1}, x_{2}\right)=\frac{\left(p_{1}^{\prime}-p_{1}\right) x_{1}+p_{2} x_{2}}{p_{2}^{\prime}} .
$$

According to (iv), $\phi\left(x^{p}(0)\right)=x^{p^{\prime}}(0)=x^{p}(0)$ and hence $\phi_{2}\left(x_{1}^{p}(0), x_{2}^{p}(0)\right)=$ $x_{2}^{p}(0)=0$. Therefore (18), for $x_{1}=x_{1}^{p}(0)$ and $x_{2}=x_{2}^{p}(0)$, implies

$$
0=\frac{\left(p_{1}^{\prime}-p_{1}\right) x_{1}^{p}(0)}{p_{2}^{\prime}} .
$$

Let us assume that $x_{1}^{p}(0)=a \neq 0$. Then $p_{1}^{\prime}=p_{1}$ and from (18) we get that

$$
\phi_{2}\left(x_{1}, x_{2}\right)=\frac{p_{2} x_{2}}{p_{2}^{\prime}} .
$$

By considering the condition (iii) for the polynomial $\varphi\left(x_{1}, x_{2}\right)=x_{2}$, by substituting $\phi_{1}\left(x_{1}, x_{2}\right)=x_{1}$ according to (ii) and $\phi_{2}\left(x_{1}, x_{2}\right)=\frac{p_{2} x_{2}}{p_{2}^{\prime}}$ according to (19), and by evaluating the received equality at the point $\left(x_{1}^{p}(0), x_{2}^{p}(0)\right)$, we derive the relation

$$
p_{1} \frac{p_{2}}{p_{2}^{\prime}} x_{1}^{p}(0)=p_{1}^{\prime} x_{1}^{p}(0)
$$

Since $p_{1}=p_{1}^{\prime}$ and $x_{1}^{p}(0)=a \neq 0$, it follows that $p_{2}=p_{2}^{\prime}$. Hence, from (19), $\phi_{2}\left(x_{1}, x_{2}\right)=x_{2}$. This together with (ii) proves that a rational mapping $\phi$ : $X^{p} \rightarrow X^{p^{\prime}}$ is the identity if it holds that $a \neq 0$. So, for $a \neq 0$, by Theorem 4.8, the parametrization $\mathcal{P}: P \rightarrow \Sigma(P)$ is structurally identifiable.

The last example treats the model of a peptide chain elongation described in [16] and consequently in [19, Chapter 8.3.3]. It is a bilinear system with parameters which is a special case of a parametrized polynomial and thus also a parametrized rational system.

Example 5.3 The model of a peptide chain elongation from [19] is given by the equations:

$$
\begin{aligned}
& \dot{B}=-k_{1} A_{i} B+k_{-1} C+k_{r} G+k_{7} F \quad(i=1,2) \\
& \dot{C}=k_{1} A_{i} B-k_{-1} C-k_{2} C+k_{-2} D \\
& \dot{D}=k_{2} C-k_{-2} D-k_{3} D \\
& \dot{E}=k_{3} D-k_{4} E \\
& \dot{F}=k_{4} E-k_{5} F-k_{7} F \\
& \dot{G}=k_{5} F-k_{r} G .
\end{aligned}
$$

The state variables $B, C, D, E, F, G$ correspond to ribosome, initial binding, codon recognition, GTPase activation and GTP hydrolysis, EF-Tu released, 
and accomodation and pepetide transfer, respectively. $A_{1}$ and $A_{2}$ stand for correct and wrong aa-tRNA which are different types of $t R N A$.

We will study the model only for one of $A_{1}, A_{2}$. We consider $A_{1}$ or $A_{2}$ to be the input $u$ of the system. We assume that the inputs $u$ are piecewise-constant functions with the values $\alpha \in \mathbb{R}$. To study structural identifiability of the model we need to specify the initial state and the outputs of the system. Let us assume that the initial state is given as $(B(0), C(0), D(0), E(0), F(0), G(0))=$ $(1,1,1,1,1,1)$ and that the outputs are given as the outflows $k_{2} C, k_{7} F$, and $k_{r} G$. Then the parametrized system modeling the elongation of a peptide chain is the structured polynomial system $\Sigma(P)$ with the parameter set $P=\mathbb{R}^{9}$ such that the polynomial systems $\Sigma(k)=\left(X^{k}, f^{k}, h^{k}, x_{0}^{k}\right) \in \Sigma(P)$ where $k=$ $\left(k_{1}, k_{-1}, k_{2}, k_{-2}, k_{3}, k_{4}, k_{5}, k_{7}, k_{r}\right) \in P$ are given as:

$$
\begin{aligned}
& X^{k}=\mathbb{R}^{6}, \\
& f_{\alpha}^{k}=\left[\left(\begin{array}{cccccc}
-k_{1} \alpha & k_{-1} & 0 & 0 & k_{7} & k_{r} \\
k_{1} \alpha & -k_{-1}-k_{2} & k_{-2} & 0 & 0 & 0 \\
0 & k_{2} & -k_{-2}-k_{3} & 0 & 0 & 0 \\
0 & 0 & k_{3} & -k_{4} & 0 & 0 \\
0 & 0 & 0 & k_{4} & -k_{5}-k_{7} & 0 \\
0 & 0 & 0 & 0 & k_{5} & -k_{r}
\end{array}\right)\left(\begin{array}{c}
B \\
C \\
D \\
E \\
F \\
G
\end{array}\right)\right]\left(\begin{array}{c}
\frac{\partial}{\partial B} \\
\frac{\partial}{\partial C} \\
\frac{\partial}{\partial D} \\
\frac{\partial}{\partial E} \\
\frac{\partial}{\partial F} \\
\frac{\partial}{\partial G}
\end{array}\right)^{T}, \\
&\left(B^{k}(0), C^{k}(0), D^{k}(0), E^{k}(0), F^{k}(0), G^{k}(0)\right)^{T}=(1,1,1,1,1,1)^{T} .
\end{aligned}
$$

Because $\mathbb{R}\left[h_{1}^{k}, h_{2}^{k}, h_{3}^{k}, f_{\alpha}^{k}\left(h_{2}^{k}\right), f_{\alpha}^{k}\left(f_{\alpha}^{k}\left(h_{2}^{k}\right)\right), f_{\alpha}^{k}\left(h_{1}^{k}\right)\right]=\mathbb{R}[B, C, D, E, F, G]$ for all $k \in P \backslash O$ where $O$ is a strict subvariety of $P$, the system $\Sigma(P)$ is structurally algebraically observable. Note that $\Sigma(P)$ would be structurally algebraically observable even if we considered only one output, namely $h^{k}=h_{3}^{k}$. Then $\mathbb{R}\left[\left\{\left(f_{\alpha}^{k}\right)^{i} h_{3}^{k} \mid i=0, \ldots, 5\right\}\right]=\mathbb{R}[B, C, D, E, F, G]$. It is easy to see that $\Sigma(P)$ is also structurally distinguishing parameters. A variety $D \subsetneq P$ such that $\Sigma(k)$ is distinguishing parameters for all $k \in P \backslash D$ can be considered to be $D=\left\{k=\left(k_{1}, k_{-1}, k_{2}, k_{-2}, k_{3}, k_{4}, k_{5}, k_{7}, k_{r}\right) \in \mathbb{R}^{9} \mid k_{1} k_{-1} k_{2} k_{-2} k_{3} k_{4} k_{5} k_{7} k_{r}=0\right\}$.

By defining new inputs as $v=B u$ we derive from the system $\Sigma(P)$ the parametrized linear system $\Sigma_{\text {lin }}(P)$. To show that $\Sigma(P)$ is structurally algebraically controllable it is sufficient to prove that $\Sigma_{\text {lin }}(P)$ is structurally controllable by checking controllability rank condition for the systems $\Sigma_{\text {lin }}(k)$ 
where $k \in P \backslash C$ for a variety $C \subsetneq P$. The system $\Sigma_{\text {lin }}(P)$ is given as

$$
\begin{aligned}
& X^{k}=\mathbb{R}^{6},
\end{aligned}
$$

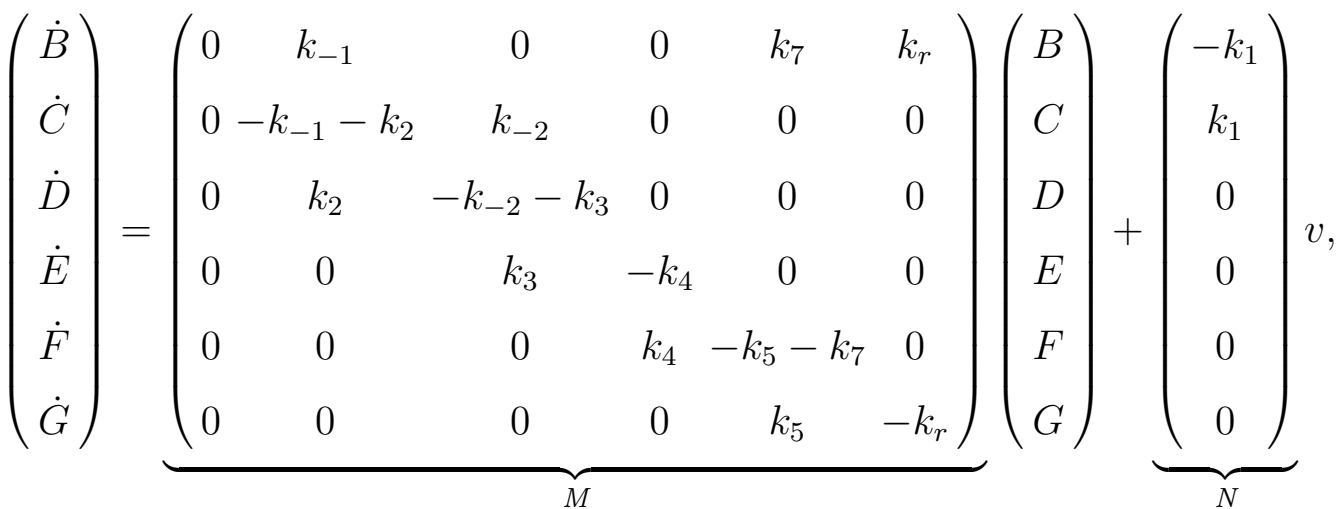

$$
\begin{aligned}
& h^{k}=\left(h_{1}^{k}, h_{2}^{k}, h_{3}^{k}\right)^{T}=\left(k_{2} C, k_{7} F, k_{r} G\right)^{T}, \\
& (B(0), C(0), D(0), E(0), F(0), G(0))^{T}=(1,1,1,1,1,1)^{T} \text {. }
\end{aligned}
$$

One can show that $\operatorname{rank}\left(N M N \ldots M^{5} N\right)=6$ for all parameters $k \in P \backslash C$ where $C$ is a strict subvariety of $P$. Therefore, the system $\Sigma(P)$ is structurally algebraically controllable and, finally, it is structurally canonical.

From the irreducibility of $P$ it follows that $O \cup C \cup D \subsetneq P$. Thus we can consider $\Sigma(k), \Sigma\left(k^{\prime}\right) \in \Sigma(P)$ such that $k, k^{\prime} \in P \backslash(O \cup C \cup D)$. Both systems are canonical and distinguishing parameters. Further, because they are realizing the same measurements, they are according to Theorem 4.2 isomorphic. From Definition 3.6, there exist polynomial mappings $\phi, \psi: \mathbb{R}^{6} \rightarrow \mathbb{R}^{6}$ such that $\phi \circ \psi=\psi \circ \phi=1_{\mathbb{R}^{6}}$ and

(i) $f_{\alpha}^{k}(\varphi \circ \phi)=\left(f_{\alpha}^{k^{\prime}} \varphi\right) \circ \phi$ for all $\alpha \in \mathbb{R}, \varphi \in \mathbb{R}\left[X_{1}, \ldots, X_{6}\right]$,

(ii) $h_{i}^{k}=h_{i}^{k^{\prime}} \circ \phi$ for $i=1,2,3$,

(iii) $\phi(B(0), C(0), D(0), E(0), F(0), G(0))=\phi(1,1,1,1,1,1)=(1,1,1,1,1,1)$.

We prove that $\phi=\left(\phi_{1}, \ldots, \phi_{6}\right)^{T}: \mathbb{R}^{6} \rightarrow \mathbb{R}^{6}$ is the identity for any $k, k^{\prime} \in$ $P \backslash S$ where $S$ is a strict subvariety of $P$ containing $C \cup O \cup D$. Then, from Theorem 4.3, the parametrization $\mathcal{P}: P \rightarrow \Sigma(P)$ defined as $\mathcal{P}(k)=\Sigma(k)$ is structurally identifiable.

If $k_{2}, k_{7}, k_{r} \neq 0$ then from (ii) it follows that $k_{2}=k_{2}^{\prime}, k_{7}=k_{7}^{\prime}, k_{r}=k_{r}^{\prime}$ and furthermore that

$$
\begin{aligned}
& \phi_{2}(B, C, D, E, F, G)=C, \\
& \phi_{5}(B, C, D, E, F, G)=F, \\
& \phi_{6}(B, C, D, E, F, G)=G .
\end{aligned}
$$


If we consider the polynomial $\varphi(B, C, D, E, F, G)=F$ in (i) and if $k_{4} \neq 0$ we derive that $k_{4}=k_{4}^{\prime}$ and moreover

$$
\phi_{4}(B, C, D, E, F, G)=E \text {. }
$$

If $k_{3} \neq 0$ then by considering the polynomial $\varphi(B, C, D, E, F, G)=E$ in (i) we get $k_{3}=k_{3}^{\prime}$ and

$$
\phi_{3}(B, C, D, E, F, G)=D .
$$

For the polynomial $\varphi(B, C, D, E, F, G)=D$ the relation (i) and the equality $k_{2}=k_{2}^{\prime}$ imply that $k_{-2}=k_{-2}^{\prime}$. Then, if $k_{1} \neq 0$, by considering the polynomial $\varphi(B, C, D, E, F, G)=C$ in (i) we derive that $\left(-k_{-1}+k_{-1}^{\prime}\right) C+k_{1} B \alpha=$ $k_{1}^{\prime} \alpha \varphi_{1}(B, C, D, E, F, G)$ for all $\alpha \in \mathbb{R}$. From (iii) follows that $-k_{-1}+k_{-1}^{\prime}=$ $\alpha\left(k_{1}^{\prime}-k_{1}\right)$ for all $\alpha \in \mathbb{R}$. Therefore $k_{-1}=k_{-1}^{\prime}, k_{1}=k_{1}^{\prime}$, and consequently

$$
\phi_{1}(B, C, D, E, F, G)=B \text {. }
$$

While deriving the equalities (20), (21), (22), and (23) we excluded only the parameters which are contained in D. Therefore $\phi$ is the identity for any $k, k^{\prime} \in P \backslash S$ where $S=C \cup O \cup D$ and the parametrization $\mathcal{P}$ is structurally identifiable.

If we consider $h_{1}^{k}=k_{1} B$ instead of $h_{1}^{k}=k_{2} C$ we can prove in the same way that the parametrization $\mathcal{P}$ is structurally identifiable.

\section{Concluding remarks}

We have shown that algebraic controllability and algebraic/rational observability are neither necessary nor sufficient conditions for structural identifiability of a parametrization of a structured polynomial or a structured rational system. The corresponding result holds also for linear systems [17].

We have provided the characterization of structural and global identifiability of parametrizations of structured polynomial and structured rational systems in terms of algebraic geometry. The basic objects we use are varieties which are determined by polynomial ideals, and the maps (on or between varieties) with which we deal are polynomial or rational. Therefore, the main results of this paper stated in Theorem 4.3, 4.8 and Remark 4.5, 4.9 make it possible to apply results of computational algebra to obtain procedures for checking structural and global identifiability of parametrizations of parametrized polynomial and parametrized rational systems. Further results concerning computational approaches to algebraic and rational observability and algebraic controllability could lead to algorithms or procedures for checking these properties. 
In this paper we assumed that the parameter sets are irreducible real affine varieties. We could also work with arbitrary subsets of $\mathbb{R}^{l}$. Then the structural properties defined in Definition 3.9 have to be considered as properties valid for all parameter values except for a set of parameter values of measure zero.

As demonstrated in [29, p. 248] and in [22, p. 14], the parametrized systems whose parametrizations are such that the rational combinations of parameters are present as coefficients in the vector fields, output functions, or initial conditions are realistic and very often necessary to faithfully describe the biological character of the studied process. Our approach allows for such parametrizations if the condition on distinguishability of parameters is satisfied.

Detailed comparison with other existing methods is worth studying as well as the problem of determining the classes of inputs which are exciting the polynomial and rational systems sufficiently well to be able to determine their identifiability. For bilinear systems, the problem of characterizing sufficiently exciting inputs is considered in [30]. The problem of structural indistinguishability should be treated as well. It deals with the uniqueness of a model structure and should be easily solvable by means of realization theory. This problem is for example treated in [14] for uncontrolled nonlinear analytic systems by generalizing the results of structural identifiability from [13]. The problem of determining the numerical values of parameters from measurements is a major open problem.

\section{Acknowledgements}

I would like to thank Jan H. van Schuppen for his advice, suggestions, and comments.

\section{References}

[1] V.I. Arnold. Ordinary differential equations. The MIT Press, 1973.

[2] J. Baillieul. The geometry of homogeneous polynomial dynamical systems. Nonlinear anal., Theory, Meth. and Appl., 4(5):879-900, 1980.

[3] J. Baillieul. Controllability and observability of polynomial dynamical systems. Nonlinear Anal., Theory, Meth. and Appl., 5(5):543-552, 1981.

[4] Z. Bartosiewicz. Rational systems and observation fields. Systems and Control Letters, 9:379-386, 1987.

[5] Z. Bartosiewicz. Minimal polynomial realizations. Mathematics of control, signals, and systems, 1:227-237, 1988. 
[6] R. Bellman and K.J. Åström. On structural identifiability. Mathematical Biosciences, 7:329-339, 1970.

[7] M.J. Chapman, K.R. Godfrey, M.J. Chappell, and N.D. Evans. Structural identifiability for a class of non-linear compartmental systems using linear/nonlinear splitting and symbolic computation. Mathematical Biosciences, 183:1-14, 1992.

[8] M.J. Chappell and K.R. Godfrey. Structural identifiability of the parameters of a nonlinear batch reactor model. Mathematical Biosciences, 108:241-251, 1992.

[9] M.J. Chappell, K.R. Godfrey, and S. Vajda. Global identifiability of the parameters of nonlinear systems with specified inputs: A comparison of methods. Mathematical Biosciences, 102:41-73, 1990.

[10] D. Cox, J. Little, and D. O'Shea. Ideals, varieties, and algorithms: An introduction to computational algebraic geometry and commutative algebra. Springer, 3rd edition, 2007.

[11] L. Denis-Vidal, G. Joly-Blanchard, and C. Noiret. Some effective approaches to check identifiability of uncontrolled nonlinear systems. Mathematics and computers in simulation, 57:35-44, 2001.

[12] L.C. Evans. Partial differential equations. Springer, 3rd edition, 2001.

[13] N.D. Evans, M.J. Chapman, M.J. Chappell, and K.R. Godfrey. Identifiability of uncontrolled nonlinear rational systems. Automatica, 38:1799-1805, 2002.

[14] N.D. Evans, M.J. Chappell, M.J. Chapman, and K.R. Godfrey. Structural indistinguishability between uncontrolled (autonomous) nonlinear analytic systems. Automatica, 40:1947-1953, 2004.

[15] T. Glad and A. Sokolov. Structural identifiability: Tools and applications. In Proceedings of the 38th Conference on Decision and Control, pages 2406-2411, Phoenix, Arizona, USA, 1999.

[16] A. Heyd and D.A. Drew. A mathematical model for elongation of a peptide chain. Bulletin of Mathematical Biology, 65:1095-1109, 2003.

[17] J.J. DiStefano III. On the relationship between structural identifiability and the controllability, observability properties. IEEE Transactions Autom. Control, 22(4):652, 1977.

[18] A. Isidori. Nonlinear control systems. Springer, 3rd edition, 2001.

[19] E. Klipp, R. Herg, A. Kowald, C. Wierling, and H. Lehrach. Systems biology in practice: Concepts, implementation and application. WILEY-VCH, Weinheim, 2005 .

[20] E. Kunz. Introduction to commutative algebra and algebraic geometry. Birkhäuser, Boston, 1985.

[21] L. Ljung and T. Glad. On global identifiability for arbitrary model parametrizations. Automatica, 30(2):265-276, 1994. 
[22] G. Margaria, E. Riccomagno, M.J. Chappell, and H.P. Wynn. Differential algebra methods for the study of the structural identifiability of rational function state-space models in the biosciences. Mathematical Biosciences, $174: 1-26,2001$.

[23] H. Nijmeijer and A. van der Schaft. Nonlinear dynamical control systems. Springer-Verlag, 1990.

[24] J. Němcová. Algebraic controllability of rational systems. submitted.

[25] J. Němcová and J.H. van Schuppen. Realization theory for rational systems: Minimal rational realizations. submitted.

[26] J. Němcová and J.H. van Schuppen. Realization theory for rational systems: The existence of rational realizations. submitted.

[27] R.L.M. Peeters and B. Hanzon. Identifiability of homogeneous systems using the state isomorphism approach. Automatica, 41:513-529, 2005.

[28] Pohjanpalo. System identifiability based on the power series expansion of the solution. Mathematical Biosciences, 41:21-33, 1978.

[29] A. Raksanyi, Y. Lecourtier, E. Walter, and A. Venot. Identifiability and distinguishability testing via computer algebra. Mathematical Biosciences, 77:245-266, 1985.

[30] E.D. Sontag, Y. Wang, and A. Megretski. Input classes for identification of bilinear systems. IEEE Transactions Autom. Control, to appear 2007.

[31] C.C. Travis and G. Haddock. On structural identification. Mathematical Biosciences, 56:157-173, 1981.

[32] S. Vajda, K.R. Godfrey, and H. Rabitz. Similarity transformation approach to identifiability analysis of nonlinear compartmental models. Mathematical Biosciences, 93:217-248, 1989.

[33] S. Vajda and H. Rabitz. State isomorphism approach to global identifiability of nonlinear systems. IEEE Transactions Autom. Control, 34(2):220-223, 1989.

[34] J.M. van den Hof. Structural identifiability of linear compartmental systems. IEEE Transactions Autom. Control, 43(6):800-818, 1998.

[35] E. Walter. In S. Levin, editor, Identifiability of state space models, volume 46 of Lecture notes in Biomathematics. Springer-Verlag, Berlin Heidelberg New York, 1982.

[36] E. Walter and Y. Lecourtier. Unidentifiable compartmental models: what to do? Mathematical Biosciences, 56:1-25, 1981.

[37] X. Xia and C.H. Moog. Identifiability of nonlinear systems with application to HIV/AIDS models. IEEE Transactions Autom. Control, 48(2):330-336, 2003.

[38] O. Zariski and P. Samuel. Commutative algebra I, II. Springer, 1958. 